\title{
Electric-Field Induced Polarization of the Layer of Condensed Ions on Cylindrical Colloids
}

\author{
Jan K.G. Dhont, Kyongok Kang \\ Forschungszentrum Jülich \\ Institute of Complex Systems (ICS-3) \\ Soft Matter \\ D-52425 Jülich \\ Germany
}

\begin{abstract}
In concentrated suspensions of charged colloids, interactions between colloids can be induced by an external electric field through the polarization of charge distributions (within the diffusive double layer and the layer of condensed ions) and/or electro-osmotic flow. In case of rod-like colloids, these field-induced inter-colloidal interactions have recently been shown to lead to anomalous orientation perpendicular to the external field, and to phase/state transitions and dynamical states, depending on the field amplitude and frequency of the external field. As a first step towards a (semi-) quantitative understanding of these phenomena we present a linear-response analysis of the frequency dependent polarization of the layer of condensed ions on a single, long and thin cylindrical colloid. The in-phase and out-phase response functions for the charge distribution and the electric potential are calculated for arbitrary orientation of the cylindrical colloid. The frequency dependent degree of alignment, which is proportional to the electric-field induced birefringence, is calculated as well, and compared to experiments on dilute fd-virus suspensions.
\end{abstract}

\section{Introduction}

Recent experiments on suspensions of charged rod-like colloids in oscillating external electric fields revealed a number of unexpected phenomena. Anomalous alignment of fd-virus particles (very long 
and thin, stiff rods) perpendicular to the external field has been found in Ref.[1] at low frequencies (below $1 \mathrm{kHz}$ ) for fd-concentrations above the overlap concentration. The present authors observed field-induced phase/state transitions, dynamical states and non-equilibrium critical behaviour in concentrated fd-virus suspensions $[2,3,4]$. A still ill-understood phenomenon is the experimentally observed crowding of spheres around rods [5] on applying an external electric field. Field-induced structures, where short ellipsoidal rods tend to align parallel to each other, have been observed in Ref.[6]. All these experiments are performed at sufficiently low frequencies (less than about a few tens of $k H z$ ) such that the double-layer charge distribution and the surface charges due to condensed ions are polarized. These polarization charges, and possibly the accompanied electro-osmotic flow, results in field-induced rod-rod interactions that are responsible for the observed phenomena.

As yet there is no theory that describes collective phenomena that originate from external fieldinduced interactions. Such a theory requires three steps. First of all, the polarization of a single rod in an oscillating electric field must be considered. Second, up to leading order in the external-field amplitude, pair-interactions can be calculated from these single-particle polarization results. As a third step, the collective behaviour should be predicted on the basis of these pair-interactions. This can not be done through a free energy route, since the system is far out-of equilibrium as far as the rod-rod interactions are concerned. Instead, kinetic equations should be derived and analyzed, which incorporate the field-induced interactions. The present paper is devoted to the first step in the development of a theory to describe the above mentioned non-equilibrium phenomena.

There are two extreme cases that can be considered for the development of an analytical theory. One extreme case is where the bare surface charge density of the rods is very small. In this case, the polarization charges are due to accumulation of ions due to the impenetrability of the colloidal core for ions. It has been shown by the present authors that the induced polarization charges are large enough to lead to strong inter-rod interactions [7]. The other extreme case is that of very high surface charge density, where a large portion of the bare charges is compensated by mobile condensed ions. In this case the field-induced interactions are mainly due to polarization of the layers of condensed ions. It is the latter case that is addressed in the present paper. The present paper aims at a description 
of the polarization of the layer of condensed ions of a single, cylindrical colloid due to an oscillating external field. This case is probably relevant for fd-virus suspensions, where approximately $80 \%$ of the immobile, bare surface charges are neutralized by condensed ions.

The polarization of the diffuse double layer that is present in solution around charged colloids is solely due to the deflection of ion fluxes, both by the core of the colloids and the electric field arising from the immobile charges on the surface of the colloidal core. Ions within the diffuse double layer are not confined, but migrate across the double layer on applying an external field. Ions are drawn into the double layer and later leave the double layer again. Contrary to ions within the diffuse double layer, condensed ions are essentially constrained to move within a layer around the core of the colloids for sufficiently weak external fields. The polarization of the layer of condensed ions is therefore very different from polarization due to the response of ions in solution, outside the layer of condensed ions. A simple model is presented, where ions are constrained to reside on the surface of a cylindrical colloid, subject to the same forces that play a role in standard electro-kinetic theory, namely the Brownian force due to concentration gradients, and the electric forces.

The discovery of ion condensation and its early development goes back to 1960-1980 (Refs.[8]-[15] are a selected number of seminal papers). The discrete line-charge model that is relevant for polyelectrolytes was introduced by Manning in Ref.[14], where a full theory for condensation phenomena is given. This theory is based on free energy considerations, where electrostatic interactions between the condensed ions play an important role. As far as we are aware, the first studies on the polarization of the charge distribution of condensed ions of a single rod-like polyelectrolyte molecule stem from Mandel [8], Oosawa [11], Manning [13] and Ray [16]. Very recently, Manning published two papers $[17,18]$ on the polarization of an assembly of oriented rods which are either parallel or perpendicular to a stationary external field. There has been no calculation yet for the polarization of condensed ions due to an oscillatory external field. In the paper by Manning in this issue [19], the oscillatory polarization of condensed ions is analyzed for the discrete polyelectrolyte line-charge distribution. The analysis is based on the free-energy that has been derived in the above mentioned references. The analysis in the present paper is for a cylindrical colloid with a continuous charge distribution, and is 
based on electro-kinetic equations of motion. Although the two approaches are quite different, the predictions of the models show remarkable similarities. There are early theories on the polarization of rod-like polyelectrolytes (a quite detailed overview can be found in Refs.[20, 21]). These theories assume an over-simplified frequency dependence of the surface conductivity: in the present paper we explicitly treat the dynamics of condensed ions, and find a frequency dependence for polarization charge densities that is very different from what is found in these earlier theories.

This paper is organized as follows. The model assumptions and the equations of motion for the charge density are introduced in section 2 . The equations of motion are solved for very long and thin cylinders in section 3, both for perpendicular and parallel orientation of the cylinder with respect to the oscillatory external field. This section also contains a discussion of numerical results. The results of our approach are compared to Manning's free-energy approach [19] in section 4. In section 5 , the electric-field induced orientational order is calculated from the Smoluchowski equation for the orientational probability density function, and a comparison with birefringence experiments on fdvirus suspensions is made. One of the assumptions in the analysis of electro-kinetic equations is that the diffuse double layer in solution as induced by the non-uniform condensate charge distribution is in instantaneous equilibrium. The validity of this assumption is discussed in section 6 . Section 7 contains conclusions and a discussion.

\section{The Model and Equations of Motion for the Charge Density and Potential}

As a simple model that probably captures the essential features of frequency-dependent polarization, the condensed ions are assumed to be confined within a thin layer surrounding the core of the rod-like colloid (as sketched in Fig.1). For low external field amplitudes, to within linear response, the ions are supposed not to be removed from this thin layer. The forces between the ions and the colloidal core that keep the ions to within the thin layer are thus assumed much larger than the force on the ions due to the external field. The layer will be assumed sufficiently thin with respect to the linear dimensions of the colloidal particle, so that the ions move essentially tangential to the surface $\mathcal{S}$ of 


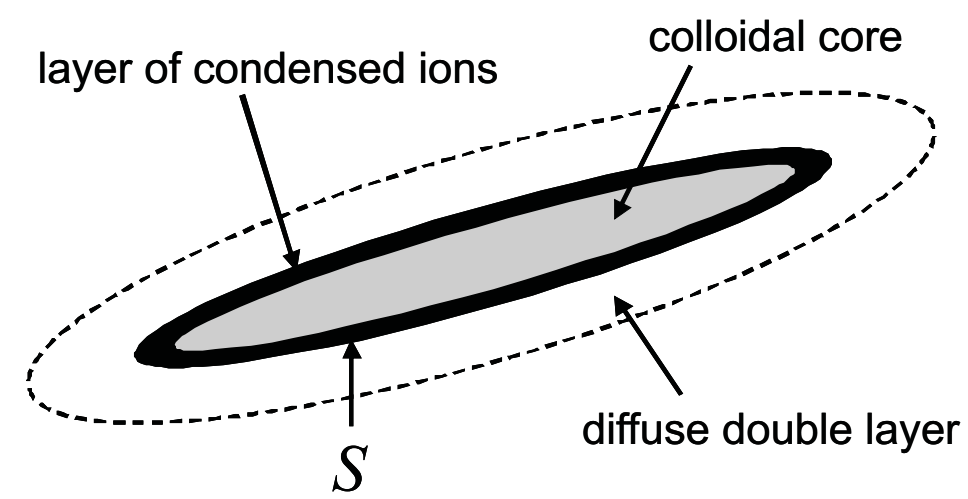

Figure 1: A sketch of the colloidal rod without an external field, where the black region at the surface is the thin layer of condensed ions. The dashed line represents the equilibrium double layer.

the core. In the mathematical limit where the thin layer is treated as a surface, the charge density is formulated in terms of a surface-charge density $\sigma$. The aim is to calculate the potential and the spatial variation of $\sigma$ over the surface $\mathcal{S}$ as induced by the external field.

That condensed ions essentially reside on the surface of macromolecules has been predicted in Ref.[14] for DNA. The prediction that condensed ions are contained within a layer of $0.7 \mathrm{~nm}$ thickness (which is close to the size of the ions) has been verified by computer simulations in Refs.[22, 23]. The same calculation as in Ref.[14], but now for fd-virus parameters, leads to a layer thickness of $0.2 \mathrm{~nm}$ [24]. These observations seem to validate the assumption in our model that the condensed ions are located right at the macromolecular surface. To within the realm of Poisson-Boltzmann theory, the spatial extent of the layer of condensed ions, including the core, is approximately equal to $\sqrt{a / \kappa}$, where $a$ is the core radius of the rod, and $\kappa^{-1}$ is the Debye length which characterizes the extent of the diffuse double layer [25]-[28]. In the experiments in Refs.[2, 3] on fd-virus suspensions, the ionic strength is quite low (Debye length is $27 \mathrm{~nm}$ ), while the radius of the rod is quite small $(3.4 \mathrm{~nm})$ as compared to other colloids. The thickness of the layer of condensed ions for this system is thus equal to $\sqrt{a / \kappa}-a=6.2 \mathrm{~nm}$. Since the condensed-ion concentration strongly increases towards the core surface, the majority of condensed ions are within a layer of a few nanometers around the core. Even for this case of quite low ionic strength and small colloidal core radius, the approximation that the ions essentially move along the core-surface seems reasonable. An analysis of the dynamics of condensed ions where the finite extent of the layer of condensed ions is accounted for would be extremely 
complicated and is probably only possible numerically. In addition, the detailed structure of the ion-concentration profile within a distance of a few nanometers from the core, besides electrostatics, also depends on solvation effects, the degree of ionization of the chemical groups that are covalently bonded to the core, ion binding, and chain-hydrophobicity (see, for example, [29]-[33]). The way in which condensation is affected by these molecular details is nowadays an active area of research. At this point it is not feasible to account for these structural details, and the present analysis is to be viewed as the simplest possible approximate theory for the dynamics of condensed ions induced by an external field. In particular we shall introduce a friction coefficient for the motion of condensed ions along the core surface, which can be very different from the friction coefficient of ions in bulk solution. Its precise value is determined by the detailed structure of the layer of condensed ions, the calculation of which is beyond the scope of the present paper.

The validity of the assumption that condensed ions are constrained to move only along the surface of the colloid may depend on the type of salt ions. From dielectric spectroscopy measurements it is known that the hydration of salt ions and their interactions with polyelectrolytes are ion-specific $[34,35]$. It is not yet known to which extent condensed ions are able to migrate in- and out-wards of the condensation region, and whether there is an ion-specificity here as well.

The assumption that the condensed ions are constrained to move within a given thin layer around the core of the rods deserves some consideration for interacting rods. As shown in Ref.[36], the extent of the condensed layer of two parallel oriented rods (without an external field) increases for intermediate separations, leading to attractive forces between the rods. It is unknown to which extent this is also important for polarized layers of condensed ions of rods which are not orientated parallel.

The total charge of the mobile condensed ions only partially compensates the bare, immobile charges on the colloid surface. The remaining, uncompensated charge resides in the diffuse double layer (represented by the dashed line in Fig.1). In the absence of an external electric field, the potential due to surface charges is screened over a distance of the order of the Debye length, due to the fact that the total charge of the core plus the condensed ions and the ions in the diffusive double layer is zero. In principle, the charge within the double layer is therefore important, since with its neglect there 
would exist an unscreened potential, also in the absence of the external field, which is unphysical. In the present treatment we will therefore assume that the majority of bare charges is compensated by condensed ions and hence, that the total charge within the diffuse double layer is relatively small. In order to prevent an unphysical unscreened contribution to the potential in the absence of the external field, we neglect the charge within the diffuse double layer, which amounts to the approximation that the total mobile charge within the condensed layer fully compensates the immobile bare charge. The constant surface-charge density of the immobile ions is denoted by $\sigma_{0}$ and that of the mobile ions by $\sigma_{m}$, so that the total surface charge density $\sigma$ is equal to,

$$
\sigma=\sigma_{0}+\sigma_{m}
$$

Within the present approach we have,

$$
\oint_{\mathcal{S}} d S \sigma_{0}(\mathbf{r}, t)=-\oint_{\mathcal{S}} d S \sigma_{m}(\mathbf{r}, t)=Q
$$

with $Q$ the total bare, immobile charge of the rod. Here, the integral ranges over the surface $\mathcal{S}$ that is supposed to contain all the mobile condensed ions as well as the immobile ions.

A realistic model for the bare charge distribution of polyelectrolytes is a line of discrete bare charges. This is a model that has been used to analyze ion condensation phenomena for polyelectrolytes at low ionic strengths $[9,11]$, which theory is commonly referred to as the "Manning condensation theory". For such a discrete line-charge distribution, the line-charge density remains finite even in case of full condensation (see also Refs.[25]-[28]). Full condensation occurs when the distance $b$ between the bare charges vanishes, that is, when the number $N$ of bare charges diverges with a fixed length $L$ of the polyelectrolyte. The number of charges that remain after condensation is $\left(b / l_{B}\right) N$, where $l_{B}$ is the Bjerrum length. The effective line-concentration is thus equal to $\left(b / l_{B}\right) N / L=\left(b / l_{B}\right)(L / b) / L=1 / l_{B}$, independent of the bare line-concentration $1 / b$. Also for full condensation there is thus a remaining finite line charge density. This result is intuitively rather obvious: when condensation took place to an extent that the remaining separation between open sites is $l_{B}$, there is no tendency for further condensation since, by definition of the Bjerrum length, no energy is gained upon further condensation. The number of mobile ions within the condensed layer is $\left(1-b / l_{B}\right) N=\left(1-b / l_{B}\right)(L / b)=L / b-L / l_{B}$ 
which diverges as $b$ vanishes. The number of ions in the diffuse double layer is equal to $\left(b / l_{B}\right) N=$ $L / l_{B}$, and is independent of $b$. The approximation in our approach is thus that the large number $\left(L / b-L / l_{B}\right)$ of condensed ions overwhelms the polarization due to the relatively few ions $\left(L / l_{B}\right)$ in the double layer that exists due to the remaining finite line-concentration $1 / l_{B}$. Furthermore, we neglect the discreteness of the bare charge distribution. These approximations are probably reasonable for large colloids with many bare charges as compared to polyelectrolytes.

The dynamics of condensed ions within the discrete line-charge distribution model for polylectrolytes is analyzed by Manning [19]. We will compare our results for a continuous charge distribution on a cylindrical colloid with Manning's results for polyelectrolytes in section 4.

For simplicity we restrict the discussion to the case where there is only a single type of mobile condensed ion. These mobile ions move under the action of two forces, which are tangential to the colloidal core since the ions are constrained to move over that surface.

First of all there is a Brownian force,

$$
\mathbf{F}^{B r}=-k_{B} T \nabla_{s} \ln c
$$

where $c$ is the surface-number concentration of mobile ions, $k_{B}$ is Boltzmann's constant and $T$ is the temperature. Furthermore, $\nabla_{s}$ is the gradient operator that is tangential to the core surface; the "surface-gradient operator" (see appendix A for the mathematical definition of the surface-gradient operator). The effects of the finite curvature can be neglected, since the radius of curvature is much larger than the linear dimensions of ions. Secondly, there is a tangential electric force,

$$
\mathbf{F}^{e}=-z e \nabla_{s} \Phi
$$

that arises from the total electric potential $\Phi$, with $z$ the valency of the condensed ions, including the sign of their charge, and $e>0$ the elementary charge.

The tangential velocity of the ions is then given by,

$$
\mathbf{v}=\frac{1}{\zeta}\left\{\mathbf{F}^{B r}+\mathbf{F}^{e}\right\}
$$

where $\zeta$ is the friction coefficient of an ion. As mentioned above, this friction coefficient may significantly differs from that of free ions in bulk solution, and depends on the detailed internal structure 
of the layer of condensed ions. Most probably the friction coefficient $\zeta$ is much larger than that for free ions. The ion fluxes are thus equal to,

$$
\mathbf{j}=\mathbf{v} c=-D\left\{\nabla_{s} c+c z e \beta \nabla_{s} \Phi\right\}
$$

where $\beta=1 / k_{B} T$, and $D=k_{B} T / \zeta$ is the diffusion coefficient of the mobile condensed ions. This is the two-dimensional analogue of the standard electro-kinetic expression for the flux. A possible convective contribution is omitted here: for incompressible fluids the effect of flow on the charge distribution is of second order in the electric field amplitude and can thus be neglected to within linear response.

There are of course also normal Brownian and electric forces acting on the mobile condensed ions. Within the model adopted here, these forces are balanced by the force arising from interactions with the colloidal core and the immobile ions on $\mathcal{S}$. This in effect assures that the ions move along the surface of the core, and implies that the condensed ions are not pealed off the surface by the external field, which is also assumed in the original theory of Manning in Ref.[15].

The expression (6) can be used in the continuity equation to obtain an explicit equation of motion for the mobile-ion concentration. The appropriate continuity equation, however, is different from the usual bulk equation, since the ions are constrained to move tangential to the surface of the colloidal core. The surface-continuity equation is derived in appendix $\mathrm{B}$, and it is shown there that the resulting diffusion equation reads,

$$
\begin{aligned}
\frac{\partial c}{\partial t} & =D \frac{1}{h_{1}}\left[\frac{\partial}{\partial s_{1}}+\frac{1}{h_{2}} \frac{\partial h_{2}}{\partial s_{1}}\right]\left\{\frac{1}{h_{1}}\left(\frac{\partial c}{\partial s_{1}}+c z e \beta\left[\frac{\partial \Phi}{\partial s_{1}}-h_{1} \mathbf{E} \cdot \hat{\mathbf{t}}_{1}\right]\right)\right\} \\
& +D \frac{1}{h_{2}}\left[\frac{\partial}{\partial s_{2}}+\frac{1}{h_{1}} \frac{\partial h_{1}}{\partial s_{2}}\right]\left\{\frac{1}{h_{2}}\left(\frac{\partial c}{\partial s_{2}}+c z e \beta\left[\frac{\partial \Phi}{\partial s_{2}}-h_{2} \mathbf{E} \cdot \hat{\mathbf{t}}_{2}\right]\right)\right\} .
\end{aligned}
$$

where the variables $s_{i}$ and the vectors $\hat{\mathbf{t}}_{i}$ have been defined in appendix B: $s_{i}$ are variables that parametrize the surface of the colloid, and $\hat{\mathbf{t}}_{i}$ are the associated, mutually orthonormal, tangential vectors.

The total potential is written as a sum of the potential $\Psi$ that arises from polarization of the condensate and that of the external electric field $\mathbf{E}$,

$$
\Phi=\Psi-\mathbf{E} \cdot \mathbf{r} .
$$


The total concentration $c$ of condensed ions is now written as the sum of the uniform surface concentration $\bar{c}$ of mobile ions that exists in the absence of the external field, and a non-uniform contribution $\Delta c$ that is induced by the external electric field,

$$
c=\bar{c}+\Delta c .
$$

Within linear response to the external field,

$$
|\Delta c / \bar{c}| \ll 1
$$

Note that the surface charged density $\sigma$, according to eqs.(1,2), is equal to,

$$
\sigma=z e \Delta c
$$

Since both $\Psi$ and $\Delta c$ are linear in the external field, eq.(7) can be linearized to within a linear-response approximation, leading to,

$$
\begin{aligned}
\frac{\partial \sigma}{\partial t} & =D \frac{1}{h_{1}}\left[\frac{\partial}{\partial s_{1}}+\frac{1}{h_{2}} \frac{\partial h_{2}}{\partial s_{1}}\right]\left\{\frac{1}{h_{1}}\left(\frac{\partial \sigma}{\partial s_{1}}+2 \epsilon \kappa_{c}\left[\frac{\partial \Psi}{\partial s_{1}}-h_{1} \mathbf{E} \cdot \hat{\mathbf{t}}_{1}\right]\right)\right\} \\
& +D \frac{1}{h_{2}}\left[\frac{\partial}{\partial s_{2}}+\frac{1}{h_{1}} \frac{\partial h_{1}}{\partial s_{2}}\right]\left\{\frac{1}{h_{2}}\left(\frac{\partial \sigma}{\partial s_{2}}+2 \epsilon \kappa_{c}\left[\frac{\partial \Psi}{\partial s_{2}}-h_{2} \mathbf{E} \cdot \hat{\mathbf{t}}_{2}\right]\right)\right\}
\end{aligned}
$$

where "the condensate-length" $\kappa_{c}^{-1}$ is defined as,

$$
\kappa_{c}=\frac{z^{2} e^{2} \beta \bar{c}}{2 \epsilon}=2 \pi z^{2} l_{B} \bar{c}
$$

with $\epsilon$ the dielectric constant of the layer of condensed ions, and $l_{B}=\beta e^{2} / 4 \pi \epsilon$ is the Bjerrum length. Since $\bar{c}$ has the dimension $m^{-2}$, it is immediately seen that $\kappa_{c}^{-1}$ has the dimension of a length. According to eqs.(1,2), the solution of the equation of motion (12) for the surface-charged density must satisfy the condition,

$$
\oint_{\mathcal{S}} d S \sigma=0
$$

The ions in solution respond to the non-uniform electric field resulting from the charge distribution within the layer of condensed ions. The ions in solution tend to form a diffuse electrical double layer with a local charge density that is opposite in sign to the local condensate charge. Here we will 


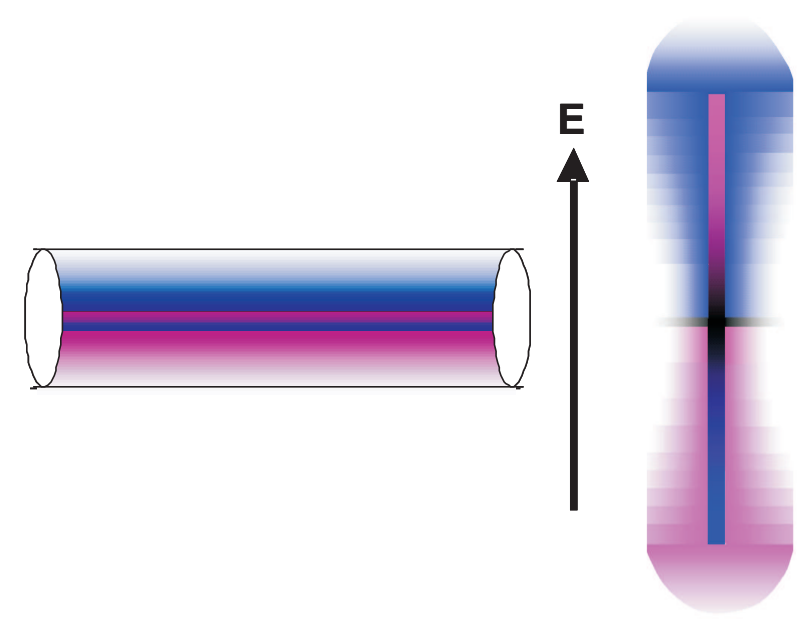

Figure 2: A sketch of the condensate-polarization-induced diffuse double layer for a rod that is aligned perpendicular (left figure) and along (right figure) the external field. Pink indicates positive charge, blue indicates negative charge. The local condensate charge is equal but opposite in sign to the charge within the surrounding double layer.

assume that the frequency is sufficiently low that the double can fully develop. This assumption will be addressed in detail in section 6 . The non-uniform double layer is sketched in Fig.2 for perpendicular and parallel orientation of the rod with respect to the external field. It is thus assumed that there is a non-uniform double layer present around the colloidal rod, which is in instantaneous equilibrium with the condensate-charge distribution. The extent of this condensate-induced double layer is equal to the Debye length $\kappa^{-1}$,

$$
\kappa=\sqrt{\frac{2 \beta z^{2} e^{2} c_{0}}{\epsilon}}
$$

where $c_{0}$ is the concentration of ions in solution outside the double layer and $\epsilon$ is the static dielectric constant of the solvent. Since the extent $\kappa^{-1}$ is independent of the local condensate charge density, the total potential due to the condensate charges and the charges within the double layer is equal to,

$$
\Psi(\mathbf{r}, t)=\frac{1}{4 \pi \epsilon} \oint_{\mathcal{S}} d S^{\prime} \sigma\left(\mathbf{r}^{\prime}, t\right) \frac{\exp \left\{-\kappa\left|\mathbf{r}-\mathbf{r}^{\prime}\right|\right\}}{\left|\mathbf{r}-\mathbf{r}^{\prime}\right|}
$$

where, as before, $\sigma$ is the surface-charge density on the core of the colloid. Note that the deformation of the condensate-induced double layer due to the external field is of second order in the field strength, since $\sigma$ itself is already first order. Within a linear response approach we can thus neglect the external field induced deformation of the double layer, in which case the potential is given by eq.(16). Since 
the double layer is in instantaneous equilibrium, the charge density $\rho_{d l}(\mathbf{r}, t)$ within the double layer, to leading order in the external electric field, is obtained by the standard expansion of the Boltzmann exponential, leading to,

$$
\rho_{d l}(\mathbf{r}, t)=\epsilon \kappa^{2} \Psi(\mathbf{r}, t) .
$$

In summary, the following approximations are made in the above discussed model:

- The charge distribution of the condensed ions on the colloid's surface is assumed continuous. The typical separation between condensed ions is thus assumed to be much less than the length scale associated with spatial variations of the density of charges. For polyelectrolytes, a discrete charge distribution would be more realistic (see the paper by Manning [19]).

- The motion of the mobile condensed ions is supposed to be restricted to the surface of the colloid. Exchange of ions between the condensed layer and the surrounding solution is thus neglected.

- The total immobile charge that is covalently bonded to the surface is supposed to be equal, but opposite in sign, to the total charge of the condensed ions. The total charge within the diffuse double layer (without the external field) is thus assumed to be relatively small as compared to the total charge of the condensed ions. This assumption is necessary to avoid unphysical unscreened contributions to the potential.

- Only a single species of mobile condensed ions is considered.

- The dielectric constant within the layer of condensed ions is taken equal to that of the pure solvent.

- The standard electro-kinetic equations are supposed to describe the motion of ions along the surface of the colloid. The friction coefficient for motion of the condensed ions along the colloidal surface can, however, be quite different from that of ions in bulk solution.

These approximations constitute the simplest possible model to analyze the polarization phenomena of the layer condensed ions. The model captures the essential features of condensate polarization, and can be employed as a starting point for the understanding of various phenomena that are found experimentally as mentioned in the introduction.

There are four mains issues that could be considered to improve the model. First of all, condensed 
ions might be able to move into the diffuse double layer and visa versa. This results in a source/sink contribution to the diffusion equation (12), and requires an additional model for the exchange kinetics. A serious approximation is the neglect of the diffuse double layer. Since the polarization of a diffuse double layer on its own (in the absence of condensed ions) is already a formidable problem, the coupled polarization of diffuse double layers and the layer of condensed ions probably requires numerical efforts, and is not amenable for analytical treatment. Thirdly, the restriction to just a single species of mobile ions could be relaxed. It turns out, however, that this complicates the analysis considerably, since it is not possible anymore to derive a closed equation of motion for the charge density. As a last possible improvement of the model, the dielectric constant of the layer of condensed ions could be accounted for. The dielectric constant within the layer of condensed ions, which is unknown, differs from that of bulk solvent due to the large concentration of condensed ions and due to the presence of a structured solvation layer.

\section{Polarization of the Condensate of Cylindrical Colloids}

The equations of motion that were formulated in the previous section will be solved here for a cylindrical colloidal rod of length $L$ and core radius $a$, with its center at the origin. The condensed ions are restricted to move along the mantle of the cylinder, so that the flat ends are uncharged. This is a good approximation for high-aspect-ratio rods, for which $a / L \ll 1$.

The field amplitude can be decomposed in components perpendicular and parallel to the long axis of the rod. To within linear response, the surface-charge density and the potential for arbitrary orientation of the rod due to both components can simply be added. We will therefore consider the two cases of rod orientation perpendicular and parallel to the electric field separately in the two following subsections.

The external electric field that is considered is spatially uniform and sinusoidally varying with time,

$$
\mathbf{E}(t)=\mathbf{E}_{0} \cos \{\omega t\}
$$

where $\omega=2 \pi \nu$ (with $\nu$ the frequency of oscillation), and where the field amplitude $\mathbf{E}_{0}$ is taken along 
the $z$-direction. The condensate surface-charge density can be written in terms of the in-phase $R_{c}^{\prime}$ and out-phase $R_{c}^{\prime \prime}$ response functions,

$$
\sigma(\mathbf{r} \mid \omega, t)=R_{c}^{\prime}(\mathbf{r} \mid \omega) \cos \{\omega t\}+R_{c}^{\prime \prime}(\mathbf{r} \mid \omega) \sin \{\omega t\}
$$

Similarly the potential can be written as,

$$
\Psi(\mathbf{r} \mid \omega, t)=P_{c}^{\prime}(\mathbf{r} \mid \omega) \cos \{\omega t\}+P_{c}^{\prime \prime}(\mathbf{r} \mid \omega) \sin \{\omega t\}
$$

with $P_{c}^{\prime}$ and $P_{c}^{\prime \prime}$ the in-phase and out-phase potential response functions, respectively. The subscript "c" refers to the condensate. The above equations of motion are most easily solved in complex form, where $\mathbf{E}(t)=\mathbf{E}_{0} \exp \{i \omega t\}$. The real, and minus the imaginary parts of the resulting complex-valued surface-charge density and potential are the in-phase and out-phase response functions, respectively.

\subsection{Perpendicular orientation}

As shown in the second part of appendix A, the surface-diffusion equation (12) for perpendicular orientation reads,

$$
\frac{\partial \sigma(\varphi, t)}{\partial t}=\frac{D}{a^{2}}\left(\frac{\partial^{2} \sigma(\varphi, t)}{\partial \varphi^{2}}+2 \epsilon \kappa_{c}\left[\frac{\partial^{2} \Psi(\varphi, y=0, \rho=a, t)}{\partial \varphi^{2}}+a E(t) \cos \varphi\right]\right) .
$$

Here, $y$ is the coordinate along the contour of the $\operatorname{rod}$ and $\varphi$ is the cylindrical azimuthal angular coordinate, where the $z$-axis is chosen along the external electric field (see Fig.9a in appendix A). Note that with the neglect of end-effects, the potential takes a value independent of $y$ as long as $\rho=a$.

The differential equations will be solved in complex form with $E(t)=E_{0} \exp \{i \omega t\}$. As mentioned above, the real and minus the imaginary parts of the resulting complex-valued $\sigma$ and $\Psi$ are the in-phase and out-phase response functions (as defined in eq.(19,20)), respectively.

To within linear response to the external field, the $\varphi$-dependence of the surface-charge density is expected to be of the form,

$$
\sigma(\varphi, t)=A \exp \{i \omega t\} \cos \varphi
$$


where $A$ is a constant, independent of $\varphi$ and time. This Ansatz will be shown to indeed solve the differential equations. Note that the Ansatz for $\sigma$ satisfies the condition (14) for an average zero charge. The potential is found from eq.(16) to be equal to,

$$
\Psi(y, \rho=a, \varphi, t)=\frac{a A \exp \{i \omega t\}}{2 \epsilon} B(y \mid \kappa a) \cos \varphi
$$

where,

$$
B(y \mid \kappa a)=\frac{1}{2 \pi \cos \varphi} \int_{0}^{2 \pi} d \varphi^{\prime} \int_{-L / 2}^{L / 2} d y^{\prime} \cos \left\{\varphi^{\prime}\right\} \frac{\exp \left\{-\kappa \sqrt{\left(y^{\prime}-y\right)^{2}+2 a^{2}\left(1-\cos \left\{\varphi^{\prime}-\varphi\right\}\right)}\right\}}{\sqrt{\left(y^{\prime}-y\right)^{2}+2 a^{2}\left(1-\cos \left\{\varphi^{\prime}-\varphi\right\}\right)}} .
$$

Using that $\cos \varphi^{\prime}=\cos \left\{\varphi^{\prime}-\varphi\right\} \cos \varphi-\sin \left\{\varphi^{\prime}-\varphi\right\} \sin \varphi$, it is readily found from symmetry that (with $\alpha=\varphi^{\prime}-\varphi$ ),

$$
B(y \mid \kappa a)=\frac{1}{2 \pi} \int_{0}^{2 \pi} d \alpha \int_{-L / 2}^{L / 2} d y^{\prime} \cos \{\alpha\} \frac{\exp \left\{-\kappa \sqrt{\left(y^{\prime}-y\right)^{2}+2 a^{2}(1-\cos \alpha)}\right\}}{\sqrt{\left(y^{\prime}-y\right)^{2}+2 a^{2}(1-\cos \alpha)}} .
$$

Since the exponent is essentially zero when $\left|y^{\prime}-y\right|$ is larger than a few times the Debye length $\kappa^{-1}$, the $y^{\prime}$-integration range can be extended to $(-\infty, \infty)$, except for $y$ within a distance of the order $\kappa^{-1}$ from the tips of the rod. For the long rods under consideration, for which $\kappa L \gg 1$, the relative contribution to the integral from the tips can be neglected. Neglecting these end-effects, $B(\kappa a) \equiv B(y \mid \kappa a)$ is thus equal to,

$$
\begin{aligned}
B(\kappa a) & =\frac{1}{2 \pi} \int_{0}^{2 \pi} d \alpha \int_{-\infty}^{\infty} d x \cos \{\alpha\} \frac{\exp \left\{-\kappa \sqrt{x^{2}+2 a^{2}(1-\cos \alpha)}\right\}}{\sqrt{x^{2}+2 a^{2}(1-\cos \alpha)}} \\
& =\frac{1}{\pi} \int_{0}^{2 \pi} d \alpha \cos \{\alpha\} K_{0}(\kappa a \sqrt{2(1-\cos \alpha)})
\end{aligned}
$$

where,

$$
K_{0}(\kappa z)=\int_{0}^{\infty} d x \frac{\exp \left\{-\kappa \sqrt{x^{2}+z^{2}}\right\}}{\sqrt{x^{2}+z^{2}}}
$$

is the modified Bessel function of the second kind of zeroth order. For small values of $\kappa a$, we can use the asymptotic form $K_{0}(x)=-\ln x$ in the integrand in eq.(26), leading to,

$$
B(\kappa a)=-\frac{1}{2 \pi} \int_{0}^{2 \pi} d \alpha \cos \{\alpha\} \ln (1-\cos \alpha)=1 .
$$




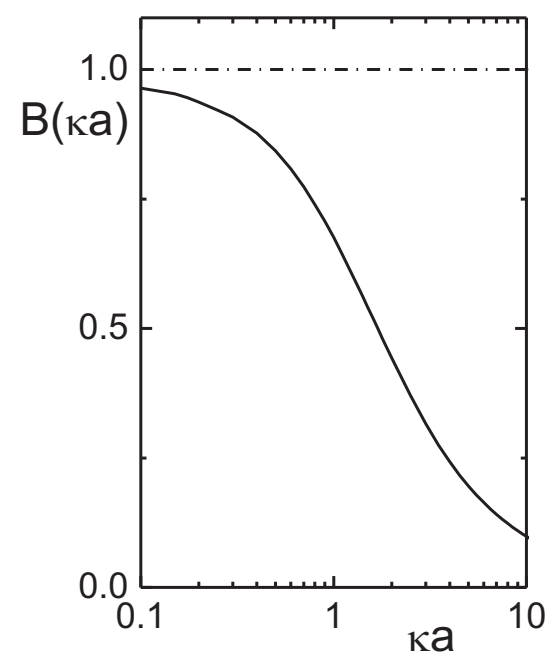

Figure 3: The function $B(\kappa a)$ in eq.(26). The solid line is obtained by numerical integration, and the dashed-dotted line is the asymptotic value 1 of $B$ for vanishing $\kappa a$.

For larger values of $\kappa a$, the integral in eq.(26) must be obtained numerically. The amplitude $B$ is plotted in Fig.3 as a function of $\kappa a$. As can be seen, the approximation (28) is reasonable for $\kappa a \lesssim 0.3-0.4$. The derivative of the potential in the equation of motion (21) is thus equal to,

$$
\frac{\partial^{2} \Psi(\varphi, y=0, \rho=a, t)}{\partial \varphi^{2}}=-\frac{a A \exp \{i \omega t\}}{2 \epsilon} B(\kappa a) \cos \varphi
$$

Substitution of this result into the equation of motion eq.(21), together with the Ansatz (22) for the surface-charge distribution immediately leads to the following expression for the amplitude $A$,

$$
A=\frac{2 \epsilon \kappa_{c} a}{1+\kappa_{c} a B(\kappa a)+i \Lambda_{\perp}} E_{0}
$$

where the dimensionless frequency $\Lambda_{\perp}$ is defined as,

$$
\Lambda_{\perp}=\frac{\omega a^{2}}{D} .
$$

This is the frequency dimensionalized by the time $a^{2} / D$ that mobile ions need to diffuse over a distance equal to the radius of the core of the rod.

The in-phase and out-phase response functions in eq.(19) are thus equal to,

$$
\begin{aligned}
R_{c, \perp}^{\prime}(\varphi \mid \omega) & =\frac{2 \epsilon \kappa_{c} a\left(1+\kappa_{c} a B(\kappa a)\right)}{\left(1+\kappa_{c} a B(\kappa a)\right)^{2}+\Lambda_{\perp}^{2}} E_{0} \cos \varphi \\
R_{c, \perp}^{\prime \prime}(\varphi \mid \omega) & =\frac{2 \epsilon \kappa_{c} a \Lambda_{\perp}}{\left(1+\kappa_{c} a B(\kappa a)\right)^{2}+\Lambda_{\perp}^{2}} E_{0} \cos \varphi
\end{aligned}
$$


where the subscript $\perp$ indicates that these response functions relate to orientation of the rod perpendicular to the field. The response functions for the potential are found from eqs. $(16,32)$ to be equal to,

$$
\begin{aligned}
& P_{c, \perp}^{\prime}(y, \rho, \varphi \mid \omega)=\frac{\kappa_{c} a^{2}\left(1+\kappa_{c} a B(\kappa a)\right)}{\left[\left(1+\kappa_{c} a B(\kappa a)\right)^{2}+\Lambda_{\perp}^{2}\right]} B(y, \rho) E_{0} \cos \varphi, \\
& P_{c, \perp}^{\prime \prime}(y, \rho, \varphi \mid \omega)=\frac{\kappa_{c} a^{2} \Lambda_{\perp}}{\left[\left(1+\kappa_{c} a B(\kappa a)\right)^{2}+\Lambda_{\perp}^{2}\right]} B(y, \rho) E_{0} \cos \varphi
\end{aligned}
$$

where,

$$
B(y, \rho)=\frac{1}{2 \pi} \int_{0}^{2 \pi} d \alpha \int_{-L / 2}^{L / 2} d x \cos \{\alpha\} \frac{\exp \left\{-\kappa \sqrt{(y-x)^{2}+a^{2}+\rho^{2}-2 a \rho \cos \alpha}\right\}}{\sqrt{(y-x)^{2}+a^{2}+\rho^{2}-2 a \rho \cos \alpha}} .
$$

The charge density inside the double layer follows from eq.(17) that connects the double-layer charge density to the potential (33). This concludes the calculation of response functions for perpendicular orientation.

\subsection{Parallel orientation}

In case the rod is oriented along the external electric field, it follows from symmetry that $\sigma$ and $\Psi$ are independent of the standard cylindrical angular variable $\varphi$, and only the dependence on $z$ remains, so that the diffusion equation (12) reduces to,

$$
\frac{\partial \sigma(z, t)}{\partial t}=D\left(\frac{\partial^{2} \sigma(z, t)}{\partial z^{2}}+2 \epsilon \kappa_{c} \frac{\partial^{2} \Psi(z, \rho=a, t)}{\partial z^{2}}\right)
$$

Here, $z$ is the coordinate along the contour of the rod, in the direction of the external field (for a precise definition of the cylindrical coordinates in this case, see Fig.9b in the second part of appendix A).

Due to the truncation of $\mathcal{S}$ at the ends of the cylinder, a boundary condition must be added that prevents the mobile ions from "leaking" at the tips of the rod into the solvent. If we would have retained the full closed form of the surface $\mathcal{S}$, such a boundary condition would not have been necessary. The flux in eq.(6) in the $z$-direction must vanish at the rod-ends, where $z= \pm \frac{1}{2} L$. From the expression (6) for the flux and eq.(81) in appendix A for the surface-gradient operator, together 
with the decomposition in eqs.(8,9) and eq.(11), leads to the boundary condition,

$$
\frac{\partial \sigma}{\partial z}+2 \epsilon \kappa_{c}\left(\frac{\partial \Psi}{\partial z}-E(t)\right)=0, \text { for } \rho=a \text { and } z= \pm \frac{1}{2} L .
$$

As before, we linearized with respect to external field strength, and the definition (13) for the condensate length $\kappa_{c}^{-1}$ has been used.

According to eq.(16) the potential within the layer of condensed ions is equal to,

$$
\Psi(Z, \rho=a, t)=\frac{a}{4 \pi \epsilon} \int_{0}^{2 \pi} d \varphi \int_{-1}^{1} d Z^{\prime} \sigma\left(Z^{\prime}, t\right) \frac{\exp \left\{-\frac{1}{2} \kappa L \sqrt{2 p^{-2}(1-\cos \varphi)+\left(Z-Z^{\prime}\right)^{2}}\right\}}{\sqrt{2 p^{-2}(1-\cos \varphi)+\left(Z-Z^{\prime}\right)^{2}}}
$$

where $Z$ is the dimensionless $z$-coordinate,

$$
Z=2 z / L
$$

and similar for $z^{\prime}$, and $p$ is the aspect ratio,

$$
p=L / 2 a \gg 1 \text {. }
$$

Since $\kappa L \gg 1$ and the aspect ratio is large, the integrand in eq.(40) is very sharply peaked around $Z^{\prime}=Z$. When the charge density does not vary significantly on length scales of the order of the Debye length $\kappa^{-1}$, the potential in eq.(40) can therefore be approximated as,

$$
\begin{aligned}
\Psi(Z, \rho=a, t) & =\frac{a}{4 \pi \epsilon} \sigma(Z, t) \int_{0}^{2 \pi} d \varphi \int_{-\infty}^{\infty} d Z^{\prime} \frac{\exp \left\{-\kappa a \sqrt{2(1-\cos \varphi)+p^{2}\left(Z-Z^{\prime}\right)^{2}}\right\}}{\sqrt{2 p^{-2}(1-\cos \varphi)+\left(Z-Z^{\prime}\right)^{2}}} \\
& =\frac{a}{\epsilon} \sigma(Z, t) K(\kappa a),
\end{aligned}
$$

except for the relatively small regions of extent $\kappa^{-1}$ near the tips of the rod. Here,

$$
K(\kappa a) \equiv \frac{1}{2 \pi} \int_{0}^{2 \pi} d \varphi K_{0}(\kappa a \sqrt{2(1-\cos \varphi)})
$$

where the integral representation (27) for the Bessel function $K_{0}$ is used. The function $K(\kappa a)$ is plotted in Fig.4 (the solid line). Like for the perpendicular orientation considered in the previous subsection, the asymptotic form $K_{0}(x)=-\ln x$ for small arguments can be used in case $\kappa a \lesssim 1$. Using that,

$$
\int_{0}^{2 \pi} d \varphi \ln \{1-\cos \varphi\}=-2 \pi \ln 2
$$


it is thus found that,

$$
K(\kappa a) \approx-\ln \{\kappa a\} \quad, \quad \kappa a \lesssim 1
$$

As can be seen from Fig.4, this approximation (the dashed-dotted line) is reasonable for $\kappa a \lesssim 0.3-0.4$.

The potential on the surface of the rod is thus found to be equal to,

$$
\Psi(Z, \rho=a, t)=\frac{a}{\epsilon} \sigma(Z, t) K(\kappa a)
$$

Substitution of eq.(44) into the dimensionless form of the diffusion equation (35) leads to the simple diffusion equation,

$$
\frac{\partial \sigma(Z, t)}{\partial t}=D^{\mathrm{eff}} \frac{\partial^{2} \sigma(Z, t)}{\partial Z^{2}}
$$

where the "effective diffusion coefficient" is equal to,

$$
D^{\mathrm{eff}}=D\left[1+2 \kappa_{c} a K(\kappa a)\right]
$$

Diffusion of condensed ions is thus enhanced by the electric field originating from the surrounding ions. Such an enhancement of concentration-gradient diffusion due to repulsive inter-particle forces is well-known for bulk systems: a gradient in concentration relaxes faster when particles repel each other. The "bare diffusion coefficient" $D$ of condensed ions can, on the contrary, be very much smaller than for free ions in solution (see the discussion in the beginning of section 2 and the comparison with experiments in section 5).

Since the boundary condition should be fulfilled just inside the charge distribution (where $\sigma \neq 0$ ), and both the potential and the charge density are smooth functions on the length scale set by the Debye length, it suffices to evaluate the integral in eq.(40), representing the potential within the layer of condensed ions, for $Z$ equal to \pm 1 minus a few times $\kappa^{-1}$. The no-flux boundary condition is thus invoked not right at the tips of the rod, but a few times the Debye length away from the tips. For large aspect ratios, this neglect of end-effects is accurate in describing the polarization of the rod. Despite the fact that eq.(44) neglects end-effects, we can therefore nevertheless use this result in the boundary condition (36), which now reads, in dimensionless form,

$$
\left[1+2 \kappa_{c} a K(\kappa a)\right] \frac{\partial \sigma(Z, t)}{\partial Z}=\epsilon \kappa_{c} L E(t) \quad, \quad \text { for } \quad Z= \pm 1
$$




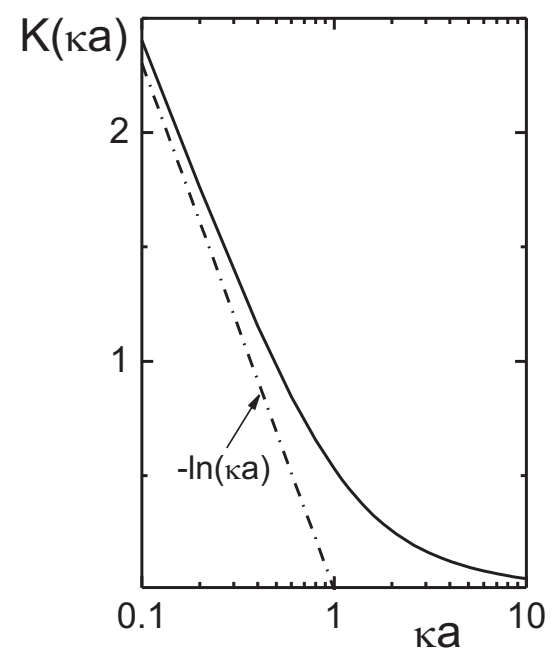

Figure 4: The function $K(\kappa a)$ in eq.(41). The solid line is obtained by numerical integration, and the dashed-dotted line is the logarithmic approximation.

The time dependence of the charge density and potential generated by the ions is made explicit as,

$$
\begin{aligned}
\sigma(Z, t) & =\sigma(Z) \exp \{i \omega t\}, \\
\Psi(Z, \rho, t) & =\Psi(Z, \rho) \exp \{i \omega t\} .
\end{aligned}
$$

Note that $\Psi$ and $\sigma$ are mutually in-phase, according to eq.(16).

The diffusion equation (45) with the boundary condition (47) are now easily solved,

$$
\sigma(Z)=\frac{\epsilon \kappa_{c} L E_{0}}{\lambda \cosh \{\lambda\}\left[1+2 \kappa_{c} a K(\kappa a)\right]} \sinh \{\lambda Z\} .
$$

where $Z$ is, as before, the dimensionless distance defined in eq.(38), and,

$$
\lambda=\frac{1+i}{\sqrt{2}} \Lambda_{\|}^{1 / 2},
$$

where $\Lambda_{\|}$is the dimensionless frequency,

$$
\Lambda_{\|}=\frac{\omega L^{2}}{4 D^{\mathrm{eff}}} .
$$

The same dimensionless frequency has been identified by Manning for polarization of the condensate on a polyelectrolyte with a discrete line-charge distribution (see the paper by Manning [19]). Note 
that the spatial variation of the charge density depends on the frequency as $\lambda \sim \omega^{1 / 2}$. This squareroot dependence for parallel orientation is to be contrasted to the analytical frequency dependence of response functions for perpendicular orientation.

As will turn out, the frequency $\omega$ where $\Lambda_{\|} \approx 1$ marks the frequency beyond which response functions begin to deviate from their stationary, zero-frequency behaviour. The frequency $\omega$ for which $\Lambda_{\|} \approx 1$ is, according to eq.(31), a factor $(L / 2 a)^{2}$ smaller than the frequency that is relevant for perpendicular orientation. The reason for this is obvious, since ions need be displaced only over distances of the order $a$ in case of perpendicular orientation, while for parallel orientation polarization occurs when ions are displaced over distances of the order $L$.

The in-phase and out-phase response functions in eq.(19) are found from eq.(49), and are most conveniently written as,

$$
\begin{aligned}
& \left(\begin{array}{c}
R_{c, \|}^{\prime}(z \mid \omega) \\
R_{c, \|}^{\prime \prime}(z \mid \omega)
\end{array}\right)=\frac{\epsilon \kappa_{c} L E_{0}}{1+2 \kappa_{c} a K(\kappa a)} \\
& \quad \times\left(\begin{array}{cc}
F^{(-)}(\Omega) & F^{(+)}(\Omega) \\
F^{(+)}(\Omega) & -F^{(-)}(\Omega)
\end{array}\right) \cdot\left(\begin{array}{c}
\cos \{2 \Omega z / L\} \sinh \{2 \Omega z / L\} \\
\sin \{2 \Omega z / L\}
\end{array}\right) .
\end{aligned}
$$

where the subscript $\|$ indicates that these response functions relate to orientation of the rod parallel to the field, where the quantity,

$$
\Omega=\left(\Lambda_{\|} / 2\right)^{1 / 2}
$$

is introduced for convenience, and,

$$
F^{( \pm)}(\Omega)=\frac{1}{\Omega} \frac{\cos \{\Omega\} \cosh \{\Omega\} \pm \sin \{\Omega\} \sinh \{\Omega\}}{\cos \{2 \Omega\}+\cosh \{2 \Omega\}} .
$$

The response functions for the potential outside the condensed layer follow from eqs. $(16,52)$,

$$
\begin{aligned}
\left(\begin{array}{c}
P_{c, \|}^{\prime}(z, \rho \mid \omega) \\
P_{c, \|}^{\prime \prime}(z, \rho \mid \omega)
\end{array}\right)=\frac{\kappa_{c} a L E_{0}}{4 \pi\left[1+2 \kappa_{c} a K(\kappa a)\right]} \\
\quad \times \int_{0}^{2 \pi} d \alpha \int_{-L / 2}^{L / 2} d x \frac{\exp \left\{-\kappa \sqrt{(z-x)^{2}+\rho^{2}+a^{2}-2 a \rho \cos \alpha}\right\}}{\sqrt{(z-x)^{2}+\rho^{2}+a^{2}-2 a \rho \cos \alpha}} \\
\quad \times\left(\begin{array}{cc}
F^{(-)}(\Omega) & F^{(+)}(\Omega) \\
F^{(+)}(\Omega) & -F^{(-)}(\Omega)
\end{array}\right) \cdot\left(\begin{array}{c}
\cos \{2 \lambda x / L\} \sinh \{2 \lambda x / L\} \\
\sin \{2 \lambda x / L\} \cosh \{2 \lambda x / L\}
\end{array}\right) .
\end{aligned}
$$

These expressions for the potential can be used in eq.(17) to obtain the charge distribution within the double layer. 

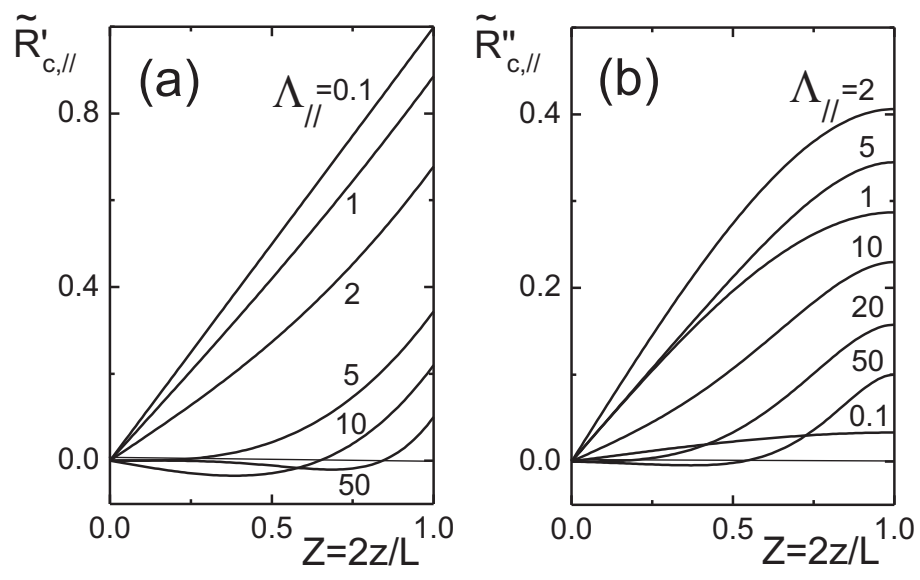

Figure 5: The in-phase (a) and out-phase (b) response functions (52) for the charge density as a function of the position along the core for various values of the dimensionless frequency $\Lambda_{\|}$, as indicated in the figures. Here, $\tilde{R}_{\|}^{\prime}=R_{\|}^{\prime} \times\left[1+2 \kappa_{c} a K(\kappa a)\right] /\left(\epsilon \kappa_{c} L E_{0, \|}\right)$, and similar for $\tilde{R}^{\prime \prime}$. Note that the response functions are odd functions of $z$, so that only the part where $z \geq 0$ is plotted.
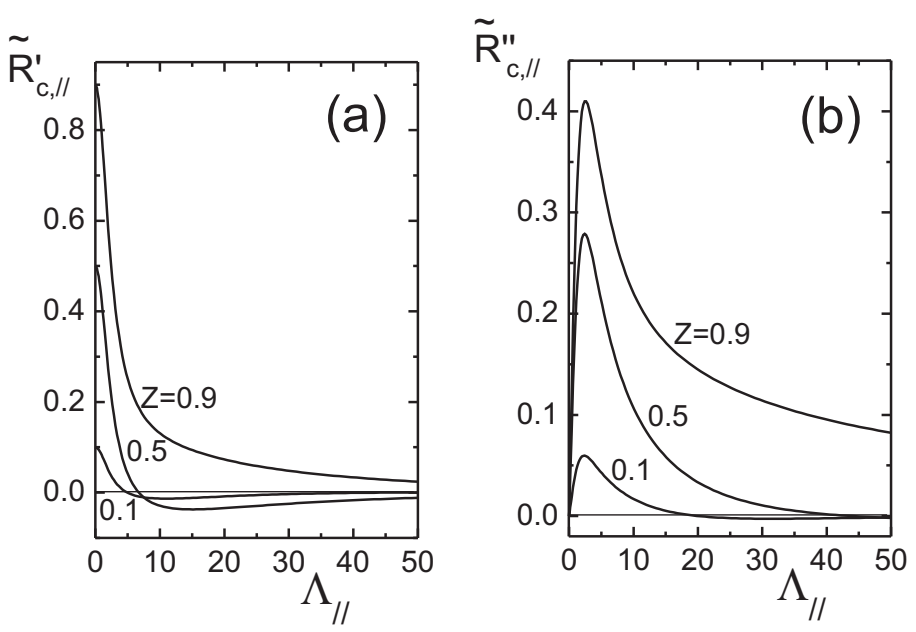

Figure 6: The in-phase (a) and out-phase (b) response functions (52) for the charge density as a function of the dimensionless frequency for three values of $Z=2 z / L$, as indicated in the figures. Like in Fig.5, $\tilde{R}_{\|}^{\prime}=R_{\|}^{\prime} \times\left[1+2 \kappa_{c} a K(\kappa a)\right] /\left(\epsilon \kappa_{c} L E_{0, \|}\right)$, and similar for $\tilde{R}^{\prime \prime}$. 
The in-phase and out-phase response functions (52) for the condensate charge density are plotted in Fig.5 as a function of $z$ for various frequencies, as indicated in the figure. For very low frequency, the in-phase charge density various linearly with $z$, while the out-phase density is zero. With increasing frequency, the in-phase density decreases in amplitude, and relatively many ions accumulate at the tips of the rod. For large frequencies the in-phase density develops a non-monotonic behaviour, where close to the origin of the rod, the charge is opposite in sign to that at the tips. At instances where the external field is directed along the positive $z$-direction, the charge at the end is positive while the charge density near the center of the rod is negative. At these high frequencies the charge density therefore exhibits a quadrupolar-like distribution. The same holds for the surrounding condensateinduced double layer, within which the charge density is, however, opposite to the condensate density. The out-phase density first increases with increasing frequency and then decreases, as expected. The frequency dependence of the charge-density response functions is shown in Fig.6, for various positions along the core of the rod, as indicated in the figure. As can be seen, the charge density at the tips of the rod is always larger as near the center. The deviation from zero-frequency response occurs at $\Lambda_{\|} \approx 1$. The response functions decay to zero at quite high values of $\Lambda_{\|}$. The reason for this is that the dimensionless number that measures the response for parallel orientation is rather $\Omega$ in eq.(53) instead of $\Lambda_{\|}$(note that for $\Lambda_{\|}=100$, at which there is essentially no response due to the finite diffusivity of the ions, the value of $\Omega=\sqrt{\Lambda_{\|} / 2} \approx 7$ ).

\section{A Comparison to Manning's Free-Energy Approach}

In the analysis of the polarization of the layer of condensed ions as given by Manning [19], ion fluxes are calculated from the free energy of the condensed charge distribution. In such an approach, the forces on ions arising from the potential set up by their inhomogeneous distribution is implicitly accounted for, so that the mathematically complicated contributions involving the potential $\Psi$ in the diffusion equation (35) and the boundary condition (36) do not appear explicitly. In Manning's approach, the core of the rod is modeled as a line with discrete bare charges of valency $z$ separated by a distance $b$. Manning derives from the free energy of condensed ions the following expression for the effective 
diffusion coefficient (in our notation) [19],

$$
D^{\mathrm{eff}}=D\left[1-2 z^{2} l_{B} \bar{c}_{l} \ln \{\kappa b\}\right],
$$

provided that $\kappa a \lesssim 1$, where $l_{B}$ is the Bjerrum length, $\bar{c}_{l}$ is the line concentration of condensed ions in the absence of the external field, that is, the number of condensed ions per unit length, and, as before, $\kappa^{-1}$ is the Debye length (see eq.(15)).

Manning's expression (56) for the effective diffusion coefficient should be compared to our expression (46). For small cylinder diameters, the approximation (43) for $K(\kappa a)$ can be used, so that eq.(46) reads,

$$
D^{\mathrm{eff}}=D\left[1-2 \kappa_{c} a \ln \{\kappa a\}\right] .
$$

Since in eq.(13) for $\kappa_{c}$, the surface concentration $\bar{c}$ is equal to $\mathcal{N} / 2 \pi a L$, with $\mathcal{N}$ the total number of condensed ions, it immediately follows that,

$$
\kappa_{c} a=z^{2} l_{B} \frac{\mathcal{N}}{L}=z^{2} l_{B} \bar{c}_{l} .
$$

The expression (56) of Manning and our expression (57) for the effective diffusion coefficient are thus identical as far as the prefactor of the logarithm is concerned. However, the argument of the logarithm in the two expressions for the effective diffusion coefficient are different: in Manning's result the distance $b$ between the discrete charges appears, while in our expression the cylinder radius $a$ appears. In fact, for the line-charge distribution in Manning's model the radius $a$ has no meaning, while in our model with a continuous charge distribution the parameter $b$ plays no role. Instead of using the free energy for a discrete line-charge distribution, the free energy of a continuous surface charge distribution renders a free energy where $b$ is replaced by $a$ [9]. This renders identical results for Manning's approach and ours.

\section{Single-Particle Alignment}

In this section we consider the alignment of a single rod in an oscillating external field. The orientational order parameter is calculated and a comparison is made with birefringence experiments on dilute fd-suspensions performed by the Konstanz group [1]. 
The electric forces with which the external field acts on the condensate are fully transferred to the rod since the condensate is constrained to move along the surface of the core. The electric forces of the external field on the condensate-induced diffuse double layer, on the contrary, are not mechanically transferred to the core of the rod. The condensate-induced double layer does, however, contribute to the total torque on the rod, through the electric forces that it exerts on the rod's surface charges.

The total torque $\mathbf{T}$ on the rod is equal to,

$$
\mathbf{T}(\hat{\mathbf{u}}, t)=\oint_{\mathcal{S}} d S \sigma(\mathbf{r}, t) \mathbf{r} \times\left[\mathbf{E}(t)-\nabla_{s} \Psi_{d l}(\mathbf{r}, t)\right]
$$

where $\Psi_{d l}$ is the potential generated by the condensate-induced double layer. The torque is a function of the unit vector $\hat{\mathbf{u}}$ along the long axis of the rod, which characterizes its orientation. The time dependence is due to the oscillating external field. For any orientation of the rod, the charge density $\sigma$ can be decomposed in its contributions $\sigma_{\|}$and $\sigma_{\perp}$ from the field components parallel and perpendicular to the rod's long axis, respectively. In this way, the torque can be written as the sum of the torque $\mathbf{T}_{\|}$that is due to forces on the charge density induced parallel to the rod's long axis and the torque $\mathbf{T}_{\perp}$ due to forces on the perpendicularly induced charge distribution,

$$
\mathbf{T}(\hat{\mathbf{u}}, t)=\mathbf{T}_{\|}(\hat{\mathbf{u}}, t)+\mathbf{T}_{\perp}(\hat{\mathbf{u}}, t)
$$

The fields that contribute to $\mathbf{T}_{\|}$are perpendicular to $\hat{\mathbf{u}}$, while the fields that contribute to $\mathbf{T}_{\perp}$ are along $\hat{\mathbf{u}}$. A long calculation leads to (mathematical details are given in appendix C),

$$
\begin{aligned}
\mathbf{T}_{\|}(\hat{\mathbf{u}}, t)= & \frac{\pi}{4} L^{3}\left(\hat{\mathbf{u}} \times \mathbf{E}_{0}\right)\left(\hat{\mathbf{u}} \cdot \mathbf{E}_{0}\right) \epsilon \\
& \times\left[F_{1} \cos \{\omega t\}+F_{2} \sin \{\omega t\}\right] \\
& \times\left[F_{3} \cos \{\omega t\}+F_{4} \sin \{\omega t\}\right],
\end{aligned}
$$

and,

$$
\begin{aligned}
\mathbf{T}_{\perp}(\hat{\mathbf{u}}, t)= & -\frac{\pi}{2} \frac{L^{3}}{p^{2}} \epsilon\left(\hat{\mathbf{u}} \times \mathbf{E}_{0}\right)\left(\hat{\mathbf{u}} \cdot \mathbf{E}_{0}\right) \\
& \times\left[H_{1} \cos \{\omega t\}+H_{2} \sin \{\omega t\}\right] \\
& \times\left[H_{3} \cos \{\omega t\}+H_{4} \sin \{\omega t\}\right],
\end{aligned}
$$


where, as before, $p$ is the aspect ratio, while,

$$
\begin{aligned}
& F_{1}=V(W+1) I^{(-)}, \\
& F_{2}=V(W+1)\left(\Omega^{-2}-I^{(+)}\right), \\
& F_{3}=2\left(1+\kappa_{c} a B(\kappa a)\right)^{2}-\kappa_{c} a\left(1+\kappa_{c} a B(\kappa a)\right)+2 \Lambda_{\perp}^{2}, \\
& F_{4}=-\kappa_{c} a \Lambda_{\perp}, \\
& H_{1}=V\left(1+W \Omega^{2} I^{(+)}\right), \\
& H_{2}=V W \Omega^{2} I^{(-)}, \\
& H_{3}=1+\kappa_{c} a B(\kappa a), \\
& H_{4}=\Lambda_{\perp} .
\end{aligned}
$$

Here, $V, W$ and $I^{( \pm)}$stand for,

$$
\begin{aligned}
V\left(\kappa_{c} a, \Lambda_{\perp}\right) & =\frac{\kappa_{c} a}{\left(1+\kappa_{c} a B(\kappa a)\right)^{2}+\Lambda_{\perp}^{2}}, \\
W\left(\kappa_{c} a, \kappa a\right) & =-\frac{2 \kappa_{c} a K(\kappa a)}{1+2 \kappa_{c} a K(\kappa a)}, \\
I^{( \pm)}(\Omega) & =\frac{1}{2 \Omega^{3}} \frac{\sinh \{2 \Omega\} \pm \sin \{2 \Omega\}}{\cosh \{2 \Omega\}+\cos \{2 \Omega\}} .
\end{aligned}
$$

The above expressions for the torque can be used to calculate the (thermally averaged) orientational order parameter that is induced in dilute suspensions, where interactions between the rods can be neglected.

The orientational order parameter, that characterizes to which extent the rods are aligned with respect to the external field, is defined as,

$$
Q_{E}(t)=\frac{3}{2}\left\{<\cos ^{2}\left\{\Theta_{E}\right\}>-\frac{1}{3}\right\}=\frac{3}{2}\left[<\hat{u}_{z}^{2}>-\frac{1}{3}\right],
$$

where, without loss of generality, the direction of the external field is chosen along the $z$-direction, $\Theta_{E}$ is the angle between $\hat{\mathbf{u}}$ and the external field, and $\hat{u}_{z}$ is the $z$-component of $\hat{\mathbf{u}}$, while the brackets $\langle\cdots\rangle$ denote ensemble averaging. For a time-independent, perfect alignment along the external field the order parameter is equal to 1 , and for perfect alignment in the plane perpendicular to the external field, the order parameter is $-1 / 2$. For a vanishing field amplitude there is an isotropic 
distribution of orientation, so that $\left\langle\hat{u}_{z}^{2}>=1 / 3\right.$, and hence $Q_{E}=0$. Therefore, when $Q_{E}<0$ the external field tends to align rods perpendicular to the external field, and when $Q_{E}>0$ there is a preferred alignment along the external field.

The orientational order parameter can be obtained from the solution of the Smoluchowski equation for the probability density function (pdf) $P(\hat{\mathbf{u}}, t)$ for the orientation $\hat{\mathbf{u}}$ of a rod, which reads,

$$
\frac{\partial}{\partial t} P(\hat{\mathbf{u}}, t)=D_{r} \hat{\mathcal{R}} \cdot[\hat{\mathcal{R}} P(\hat{\mathbf{u}}, t)-\beta P(\hat{\mathbf{u}}, t) \mathbf{T}(\hat{\mathbf{u}}, t)],
$$

where $D_{r}$ is the single-particle rotational diffusion coefficient, and $\hat{\mathcal{R}}$ is the "orientational operator",

$$
\hat{\mathcal{R}}(\cdots)=\hat{\mathbf{u}} \times \nabla_{\hat{\mathbf{u}}}(\cdots),
$$

with $\nabla_{\hat{\mathbf{u}}}$ the gradient operator with respect to the Cartesian coordinates of $\hat{\mathbf{u}}$. In terms of the pdf $P(\hat{\mathbf{u}}, t)$, the order parameter is equal to,

$$
Q_{E}(t)=\frac{3}{2} \oint d \hat{\mathbf{u}}\left[\hat{u}_{z}^{2}-\frac{1}{3}\right] P(\hat{\mathbf{u}}, t)
$$

where the integral ranges over all directions of $\hat{\mathbf{u}}$.

Multiplying both sides of the orientational diffusion equation (66) by $\hat{u}_{z}^{2}$, integrating over all directions of $\hat{\mathbf{u}}$, and performing a partial integration by means of Stokes's theorem, leads to,

$$
\frac{d}{d t} Q_{E}(t)=-6 D_{r}\left\{\left[1-\frac{1}{3} \beta E_{0}^{2} T(t)\right] Q_{E}(t)-\frac{1}{6} \beta E_{0}^{2} T(t)\left[1-3<\hat{u}_{z}^{4}>(t)\right]\right\} .
$$

where it is used that (with $\hat{u}_{x}$ and $\hat{u}_{y}$ the $x$ - and $y$-components of $\hat{\mathbf{u}}$, respectively),

$$
\begin{aligned}
\hat{\mathcal{R}} \cdot \hat{\mathcal{R}} \hat{u}_{z}^{2} & =-6 \hat{u}_{z}^{2}+2, \\
\hat{\mathcal{R}} \hat{u}_{z}^{2} & =2 \hat{u}_{z}\left(\hat{u}_{y},-\hat{u}_{x}, 0\right) .
\end{aligned}
$$

Furthermore, $T$ is defined as,

$$
\mathbf{T}(\hat{\mathbf{u}}, t)=T(t)\left(\hat{\mathbf{u}} \times \mathbf{E}_{0}\right)\left(\hat{\mathbf{u}} \cdot \mathbf{E}_{0}\right)=E_{0}^{2} T(t) \hat{u}_{z}\left(\hat{u}_{y},-\hat{u}_{x}, 0\right),
$$

for which an explicit expression immediately follows from eqs.(60-64),

$$
T(t)=T_{\|}(t)+T_{\perp}(t),
$$




$$
\begin{aligned}
& T_{\|}(t)=\frac{\pi}{4} L^{3} \epsilon {\left[F_{1} \cos \{\omega t\}+F_{2} \sin \{\omega t\}\right] } \\
& \times {\left[F_{3} \cos \{\omega t\}+F_{4} \sin \{\omega t\}\right], } \\
& T_{\perp}(t)=-\frac{\pi}{2} \frac{L^{3}}{p^{2}} \epsilon\left[H_{1} \cos \{\omega t\}+H_{2} \sin \{\omega t\}\right] \\
& \times\left[H_{3} \cos \{\omega t\}+H_{4} \sin \{\omega t\}\right] .
\end{aligned}
$$

To within linear response theory, the right hand-side of eq.(69) is expanded to leading order in $E_{0}^{2}$. Hence, for the terms in eq.(69) that are multiplied by $E_{0}^{2}$ we can use their isotropic values, as if there is no external field. Since $Q_{E}=0$ in the absence of the external field, the term $\sim E_{0}^{2} Q_{E}$ is of higher order, and is therefore neglected. Furthermore, the isotropic average of $\hat{u}_{z}^{4}$ is equal to $1 / 5$, so that we finally find the following equation of motion for the orientational order parameter,

$$
\frac{d}{d t} Q_{E}(t)=-6 D_{r}\left\{Q_{E}(t)-\frac{1}{15} \beta E_{0}^{2} T(t)\right\}
$$

the solution of which reads,

$$
Q_{E}(t)=\frac{2}{5} \beta D_{r} E_{0}^{2} \int_{-\infty}^{t} d \tau T(\tau) \exp \left\{-6 D_{r}(t-\tau)\right\}
$$

Substitution of the expressions (59-64) and performing the $\tau$-integration leads to an explicit expression for the order parameter, which is given in appendix D. In this rather complicated expression, there are three time scales of interest: the time $1 / D_{r}$ on which the rod performs a Brownian rotation, and the two times $a^{2} / D$ and $L^{2} / 4 D^{\mathrm{eff}}$ on which perpendicular and parallel polarization occurs. The rotational time scale is by far the longest (for fd virus $1 / D_{r} \approx 0.05 s$ ). For frequencies where $\omega / D_{r} \gg 1$, the rotational dynamics of the rod is not probed, but the remaining two dimensionless groups $\Lambda_{\perp}=\omega a^{2} / D$ and $\Lambda_{\|}=\omega L^{2} / 4 D^{\mathrm{eff}}$ (or, alternatively, $\Omega$ ) can be small for such frequencies. Since experiments are usually performed at frequencies where $\omega / D_{r} \gg 1$ (for fd virus $\omega / D_{r} \approx 1$ for $\nu=3 H z$ ), we will only consider the corresponding limiting form of the generally valid expression for the order parameter in eqs.(123-125) in appendix D. For $\omega / D_{r} \gg 1$, the orientational order parameter is found from eqs.(123-125) to be equal to,

$$
Q_{E}=Q_{\|}+Q_{\perp}
$$



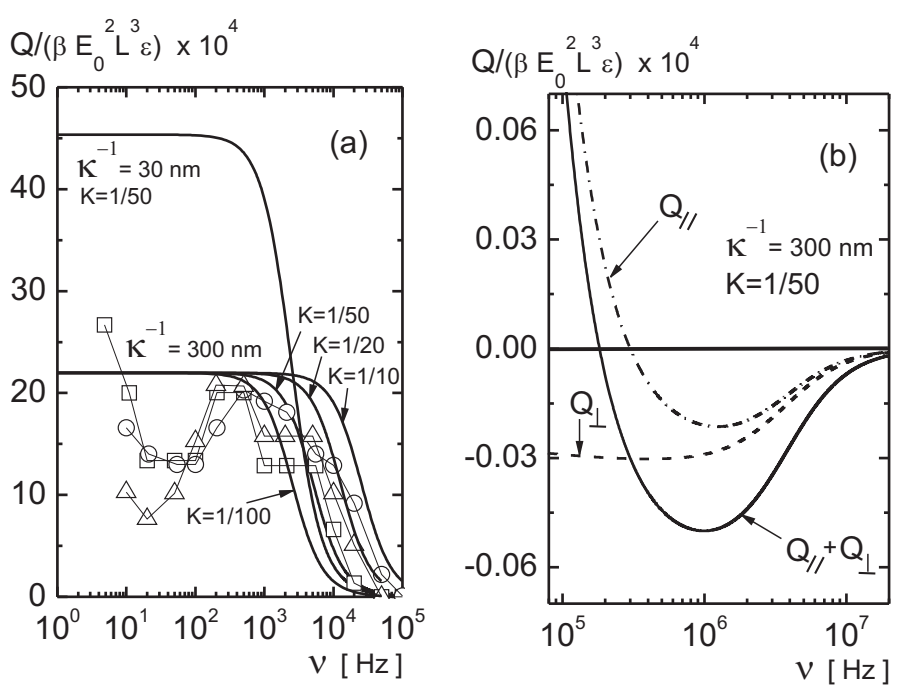

Figure 7: The order parameter $Q_{E}$ in units of $\beta E_{0}^{2} L^{3} \epsilon$ as a function of the frequency $\nu=\omega / 2 \pi$. In (a) the order parameter is shown as a function of frequency for two Debye lengths, as indicated in the figure. In (b) the order parameter for large frequencies is plotted, where it can be seen that there is a tendency to align perpendicular to the external field. In (a) theoretical plots are given for $K=1 / 100,1 / 50,1 / 20$ and $1 / 10$ in case of $\kappa^{-1}=300 \mathrm{~nm}$, and for $K=1 / 50$ in case of $\kappa^{-1}=30 \mathrm{~nm}$, while in (b) $K=1 / 50$. In (a), the data points $\square$ are for $c=0.35 c^{\star}, E_{0}=12 \mathrm{~V} / \mathrm{mm}$, the $\circ$ for $0.45 c^{\star}, E_{0}=24 \mathrm{~V} / \mathrm{mm}$ and $\Delta$ for $c=0.45 c^{\star}, E_{0}=12 \mathrm{~V} / \mathrm{mm}$, as taken from Figs.8 and 9 in Ref.[1].

with,

$$
\begin{aligned}
Q_{\|} & =\frac{\pi}{120} \beta E_{0}^{2} L^{3} \epsilon\left\{F_{1} F_{3}+F_{2} F_{4}\right\}, \\
Q_{\perp} & =-\frac{\pi}{60 p^{2}} \beta E_{0}^{2} L^{3} \epsilon\left\{H_{1} H_{3}+H_{2} H_{4}\right\} \quad, \quad \omega / D_{r} \gg 1,
\end{aligned}
$$

where, as before, the indices $\|$ and $\perp$ refer to the contributions arising from the torque on the charge distribution along and perpendicular to the rod's long axis, respectively, and the frequency dependent functions $F_{i}$ and $H_{i}$ are given in eq.(63). Note that there is no time-dependencies in eqs.(75,76), which is due to the fact that the orientation of the rod can not respond to the external field for frequencies where $\omega / D_{r} \gg 1$. The orientational order parameter is thus time-independent, despite an oscillating external field. For lower frequencies the rod is able to re-orient, resulting in an explicit time dependence of the order parameter, which is described by the full expression (123-125) in appendix D. 
The above predictions can be compared to birefringence experiments that have been performed on fd-virus suspensions in Ref.[1]. For fd-virus particles, the radius of the core is $3.4 \mathrm{~nm}$, and its length is $880 \mathrm{~nm}$, while the number of bare charges per $\mathrm{nm}$ is about 10. Since the Bjerrum length for water is $0.7 \mathrm{~nm}$, it follows from eq.(58) that $\kappa_{c} a=7$. The ionic strength in Ref.[1] is stated to be $10^{-6} M$, corresponding to a Debye length of $\kappa^{-1}=300 \mathrm{~nm}$, so that $\kappa a=0.011$. The diffusion coefficient of salt ions in bulk solution is typically $2 \times 10^{-9} \mathrm{~m}^{2} / \mathrm{s}$. The diffusion coefficient for the condensed ions may be quite different. We therefore introduce a factor $K$, that sets the diffusion coefficient to $2 \times K \times 10^{-9} \mathrm{~m}^{2} / \mathrm{s}$, that is, the friction coefficient for condensed ions is $1 / K$ times the friction coefficient of ions in bulk water. This parameter is the only fitting parameter in comparing experiment with theory.

The order parameter is related to the birefringence $\Delta n$ as,

$$
Q_{E}=\Delta n / \overline{\Delta n},
$$

where $\overline{\Delta n}$ is the maximum birefringence at the given concentration $c$ for perfectly aligned rods. For fd-viruses,

$$
\overline{\Delta n}=3.8 \times 10^{-5} \times c[\mathrm{mg} / \mathrm{ml}]
$$

The proportionality constant is taken from Ref.[37]. There is different value for the proportionality constant reported in Ref.[38] which is too large, and leads to unphysical values of the order parameter for nematic suspensions of fd-virus in equilibrium [39]. The lowest fd-concentrations considered in Ref.[1] are $c=0.35 c^{\star}$ and $0.45 c^{\star}$ (with $c^{\star}=0.04 \mathrm{mg} / \mathrm{ml}$ the overlap concentration, defined as $1 / L^{3}$ ), and birefringence experiments have been done at field strengths of $E_{0}=12 \mathrm{~V} / \mathrm{mm}$ and $24 \mathrm{~V} / \mathrm{mm}$. For these values of the field amplitude, $\beta E_{0}^{2} L^{3} \epsilon=16.4$ and 65.6 , respectively, so that eqs. $(77,78)$ can be rewritten as,

$$
\begin{aligned}
\frac{Q_{E}}{\beta E_{0}^{2} L^{3} \epsilon} & =4.0 \times 10^{4} \times \Delta n \times \frac{c^{\star}}{c}, \quad E_{0}=12 \mathrm{~V} / \mathrm{mm} \\
& =1.0 \times 10^{4} \times \Delta n \times \frac{c^{\star}}{c}, \quad E_{0}=24 \mathrm{~V} / \mathrm{mm} .
\end{aligned}
$$

Orientational order parameters as obtained from the above theory, using the fd-virus parameters, are plotted as a function of the frequency $\nu=\omega / 2 \pi$ in Fig.7. The data points in Fig.7a are calculated 
with eq.(79) from Figs.8 and 9 in Ref.[1] (their $\Delta n_{\max }$ is our $\Delta n$ ). For the Debye length of $\kappa^{-1}=$ $300 \mathrm{~nm}$, theoretical plots are given in Fig.7a for four values of $K: 1 / 100,1 / 50,1 / 20$ and 1/10. A reasonable comparison with experimental data is found when $K$ is in between $1 / 50$ and $1 / 20$. That is, the friction coefficient of the condensed ions is about 20 to 50 times larger than for ions in bulk solution. This rather large value of the friction coefficient might be due to the fact that the surface of fd-virus consists of coat proteins, resulting in a rather rough surface.

For the two fd-concentrations of $c=0.35 c^{\star}$ and $0.45 c^{\star}$, interactions between $\mathrm{fd}$ viruses will play a role. These interactions are responsible for the "dip" that is found in the measured birefringence in the frequency range $10-100 \mathrm{~Hz}$ (see the data points in Fig.7a). This dip becomes more pronounced at higher concentrations, and leads to a sign change of the measured birefringence for concentrations above $c^{\star}[1]$. At these low frequencies and relatively high concentrations the fd viruses preferentially orient perpendicular to the external field for reasons that are not yet understood. In our present calculations such interaction effects are absent, so that this dip is not observed in the theoretical curves in Fig.7a.

We note that the birefringence as plotted in the experimental graphs in Ref.[1], is the contribution that we consider here plus the amplitude of the oscillating contribution (see eqs.(123-125) in appendix D). For frequencies larger than $\sim 30 \mathrm{~Hz}$, for which $\omega / D_{r}$ is large, the oscillation part can be neglected. For lower frequencies, below $\sim 30 \mathrm{~Hz}$, the amplitude of the oscillating contribution to the order parameter might come into play.

At quite high frequencies (larger than about $2 \times 10^{5} \mathrm{~Hz}$ ) there is an interesting behaviour of the order parameter, as can be seen from Fig.7b. The contribution $Q_{\perp}$ due to perpendicular polarization is always negative, and thus leads to a preferred orientational orientation perpendicular to the external field, which is intuitively obvious. The contribution $Q_{\|}$is positive for the same obvious reasons, except for high frequencies (see the dashed-dotted line in Fig.7b). The interpretation is that at these high frequencies the induced charge density exhibits a large out-of-phase component with the external field, leading to a dipole moment that is opposite to the external field. This can be seen from Fig.8, where the in-phase (solid lines) and out-phase (dashed lines) for the dipole moment connected to the 

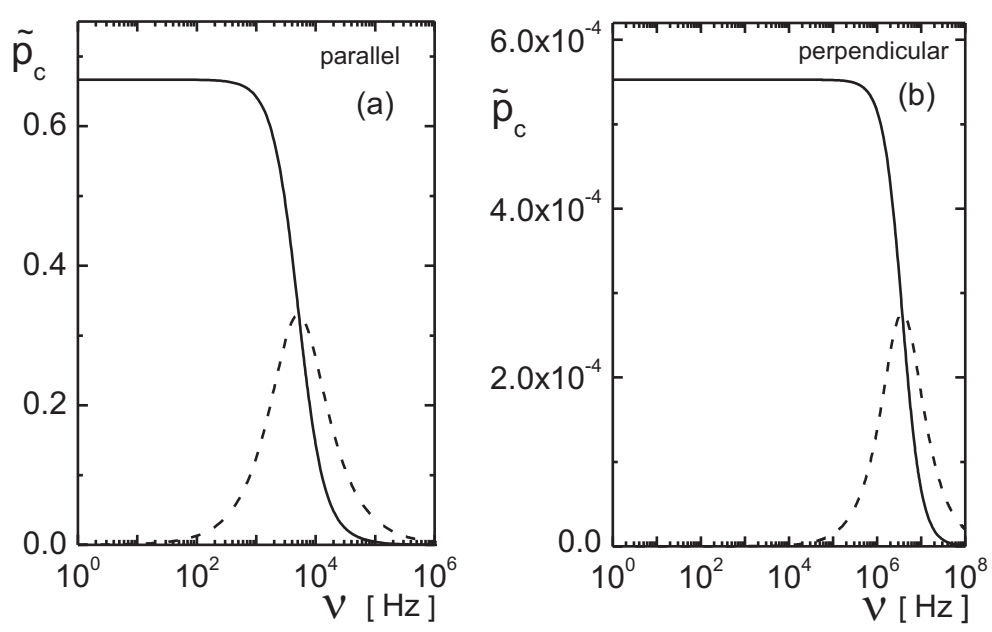

Figure 8: The dipole moments of the condensate (without the contribution from the diffuse double layer) as a function of frequency, where $\tilde{p}_{c}=p_{c} \times \frac{\pi}{2} \epsilon \kappa_{c} a L^{3} E_{0} /\left[1-2 \kappa_{c} a \ln \{\kappa a\}\right]$, where $p_{c}$ is the dipole moment of the condensate. The parameters are the same as in Fig.7, with $K=1 / 50$ and $\kappa^{-1}=300 \mathrm{~nm}$. (a) is for the dipole due to polarization parallel to the rod's long axis and (b) for perpendicular polarization. The solid lines are the in-phase components and the dashed lines to the out-phase components.

condensate charge parallel (a) and perpendicular (b) to the rod's long axis are plotted. The out-phase component becomes larger than the in-phase component at sufficiently high frequencies, which leads to the above mentioned inversion of the order parameter. Note that the inversion frequency is much larger for perpendicular orientation and that the perpendicular dipole moment is typically a factor $p^{-2} \approx 10^{-5}$ smaller than the parallel dipole moment. At these high frequencies the internal energy is decreased by orientation of the rod perpendicular to the field. Note, however, that the corresponding order parameter is very small as compared to lower frequencies, so that for fd virus suspensions this effect can not be detected experimentally.

At higher concentrations, alignment is affected by the interactions between the rods through the induced polarization charges, both of the condensate and the condensate-induced diffuse double layer. The above mentioned anomalous alignment in concentrated fd-virus suspensions perpendicular to the external field [1] is the result of such rod-rod interactions. The same interactions are at the origin of the field-induced phases and dynamical states found for fd-virus suspensions in Refs.[2, 3]. The field-induced polarization-charge interactions between rods can in principle be calculated from the 
results obtained in the present paper, which is a subject of current work.

\section{The Equilibrium Diffuse Double Layer}

It has been assumed in our analysis that there is a condensate-induced diffuse double layer in solution that is in equilibrium with the instantaneous condensate charge distribution. This is a good approximation when the time period $1 / \nu$ of oscillation of the external field (with $\nu=\omega / 2 \pi$ its frequency) is large compared to the time $1 /\left(2 D_{0} \kappa^{2}\right)$ that ions need to diffuse over a distance equal to the Debye length $\kappa^{-1}$ (where $D_{0}$ is the diffusion coefficient for ions in bulk water). The Debye length is the appropriate length scale, since the diffuse double layer is build up most rapidly through diffusion of ions perpendicular to the rod axis. Additional fluxes, along the long axis of the rod, will diminish the time scale on which a diffuse double layer is formed. A conservative condition for the presence of an equilibrium diffuse double layer is thus,

$$
\frac{\nu}{2 D_{0} \kappa^{2}} \ll 1
$$

For such low frequencies the condensate-induced double layer will fully develop, as if it were in equilibrium with the instantaneous condensate charge distribution. Since typical diffusion coefficients of ions are of the order $2 \times 10^{-9} \mathrm{~m}^{2} / \mathrm{s}$, and Debye lengths for aqueous systems typically vary from a few nanometers to about $100 \mathrm{~nm}$, the condition (80) is fulfilled for frequencies $\nu$ less than $\sim 10^{5} \mathrm{~Hz}$.

The response functions for perpendicular polarization begin to develop an out-phase component for frequencies such that $\Lambda_{\perp} \approx 1$, that is, $\omega \approx D / a^{2}$. According to the condition (80), the perpendicular orientation can therefore exhibit an out-phase component for perpendicular orientation in the presence of an equilibrium double layer when $\left(D / D_{0}\right)(\kappa a)^{2} \ll 1$. On the basis of the comparison with experiments on fd-virus suspensions in section 5 we found that $D / D_{0} \approx 1 / 50$ to $1 / 20$. One might therefore conclude that the Debye length must be of the order of, or less than, the rod-core radius in order that our theory for perpendicular polarization is valid. However, the analysis for perpendicular orientation remains valid for all frequencies for Debye lengths that are larger than the core radius a. This is due to the fact that interactions between condensed ions along the circumference of the cylinder are not affected by the double layer when it is much more extended than the core of the rod. 
Formally this is expressed by the fact that the amplitude $B$ in eq.(23) becomes independent of the Debye length for $\kappa a \lesssim 0.3-0.4$ (see Fig.3). For such large Debye lengths, the presence of a double layer is of no consequence for the perpendicular charge distribution. The potential response functions $(33,34)$ for perpendicular polarization, however, are affected by the double layer for perpendicular distances of the order $\kappa^{-1}$ from the core. Contrary to perpendicular orientation, the presence of an equilibrium double layer remains important for parallel orientation also for large Debye lengths (for which $\kappa a \lesssim 1$ ), provided that the Debye length is short as compared to the length of the rod.

We note that the numerical results in Fig.7b are somewhat beyond the validity of the condition (80) for the presence of an equilibrium double layer. The principal effect demonstrated in this figure remains valid (namely, the development of an out-phase component of the parallel charge distribution, leading to an anomalous orientation perpendicular to the external field). For smaller Debye lengths the same anomalous orientation is found within a range of frequencies where the condition (80) is satisfied.

\section{Summary and Conclusions}

We analyzed the polarization of the layer of condensed ions on cylindrical colloids under oscillatory external electric fields, within linear response. The condensed ions are assumed to migrate over the surface of the cylinder. The theory also applies to surface-conductive ions, other than condensed ions. Polarization for perpendicular and parallel orientation is analyzed, from which the polarization for arbitrary rod-orientation can be obtained by decomposing the external field into its components along and perpendicular to the rod's long axis. The in- and out-phase response functions for both the charge distribution and the potential are calculated. The non-uniform charge distribution of condensed ions induces a diffuse double layer in solution. A criterion is discussed for the existence of an instantaneous double-layer charge distribution that is in quasi-equilibrium with the condensate. For Debye lengths that are large compared to the radius of the cylindrical core, the existence of a double layer is of no consequence for the perpendicular charge distribution. For parallel polarization there is an effective diffusion coefficient which is a function of the Debye length due to screened interactions between the 
condensed ions.

A comparison is made between our model, where the charge distribution on the cylindrical core is assumed to be continuous, and a model for polyelectrolytes where a discrete bare line-charge distribution is assumed and a free-energy approach is taken (see the paper by Manning [19]). It turns out that there are striking similarities between the predictions of both models. The equations of motion for the parallel charge distribution turn out to be essentially the same, except that the distance $b$ between the discrete charged in the polyelectrolyte model that occurs in the expression for the effective diffusion coefficient is the core radius $a$ in our model. However, if one uses the free energy of a continuous charge distribution of a cylinder with a large Debye length, instead of the discrete charge distribution, the parameter $b$ is found to be replaced by $a$. The free-energy approach as developed by Manning thus renders the same expression for the effective diffusion coefficient as we find in our approach based on electro-kinetic equations.

The orientational order parameter of very dilute suspensions is calculated from the Smoluchowski equation for the orientational probability density function. A reasonable comparison with experiments on fd-virus suspensions is obtained when the friction coefficient of the condensed ions is taken a factor 20 to 50 larger than that of free ions in bulk water. At relatively high frequencies, the torque on the parallel induced charge distribution changes sign, leading to anomalous orientation perpendicular to the external field. This sign change is due to the large out-phase component of the induced dipoles.

The motivation for this work comes from the experiments on concentrated fd-virus suspensions that show unexpected phases and dynamical states depending on the frequency and external field amplitude. The necessary theoretical steps to be taken in order to understand the observed phase/state behaviour in electric fields are (i) to calculate the pair-interactions between the rods due to polarization charges, (ii) to asses the importance of interactions through electro-osmotic flow, and (iii) to analyze equations of motion for concentration and orientational order to predict collective behaviour on the basis of pair-interactions. These theoretical steps will be discussed in a separate paper based on the present analysis. 


\section{Acknowledgement}

This work has significantly benefitted from many discussions with Prof. G.S. Manning.

Part of this research was supported by the Trans-regio SFB 6018 "Physics of Colloidal Dispersions in External Fields" and by the EU-FP7 Network "NanoDirect" (contract number CP-FP-213948-2).

\section{Appendix A: The surface-gradient operator and coordi- nate systems for perpendicular and parallel orientation}

The mathematics is most conveniently formulated in terms of a parametrization $\left(s_{1}, s_{2}, s_{3}\right)$, where for a given value of $s_{3}$ the remaining two variables $s_{1}$ and $s_{2}$ parameterize the surface $\mathcal{S}$ of the core, that is, any $\mathbf{r} \in \mathcal{S}$ can be written as $\mathbf{r} \equiv \mathbf{r}\left(s_{1}, s_{2}, s_{3}=R\right)$, where the given value $R$ of $s_{3}$ sets the size of the colloidal core. The surface-gradient operator acting on an arbitrary function $f$, in terms of such a parametrization, reads,

$$
\nabla_{s} f=\frac{1}{h_{1}} \hat{\mathbf{t}}_{1} \frac{\partial f}{\partial s_{1}}+\frac{1}{h_{2}} \hat{\mathbf{t}}_{2} \frac{\partial f}{\partial s_{2}},
$$

where,

$$
\hat{\mathbf{t}}_{i}=\frac{1}{h_{i}} \frac{\partial \mathbf{r}\left(s_{1}, s_{2}, s_{3}=R\right)}{\partial s_{i}}
$$

are unit vectors, tangential to $\mathcal{S}$ (for $i=1$ and 2), with,

$$
h_{i}=\left|\frac{\partial \mathbf{r}\left(s_{1}, s_{2}, s_{3}=R\right)}{\partial s_{i}}\right| .
$$

The parametrization is assumed such that $\hat{\mathbf{t}}_{1} \perp \hat{\mathbf{t}}_{2}$, and the unit vectors are chosen such that $\hat{\mathbf{t}}_{3}=$ $\hat{\mathbf{t}}_{1} \times \hat{\mathbf{t}}_{2}$ is the unit normal on $\mathcal{S}$ that points outwards of the core (which complies with a right-handed coordinate system).

For an orientation of the rod perpendicular to the external field, we use cylindrical coordinates as defined in Fig.9a. These are the standard cylindrical coordinates except that the axis are interchanged. Here, $s_{1}=\varphi, s_{2}=y$ and $s_{3}=\rho$, while $\mathbf{r}=(\rho \sin \varphi, y, \rho \cos \varphi)$. Hence, from eqs. $(82,83)$,

$$
\hat{\mathbf{t}}_{1}=(\cos \varphi, 0,-\sin \varphi),
$$




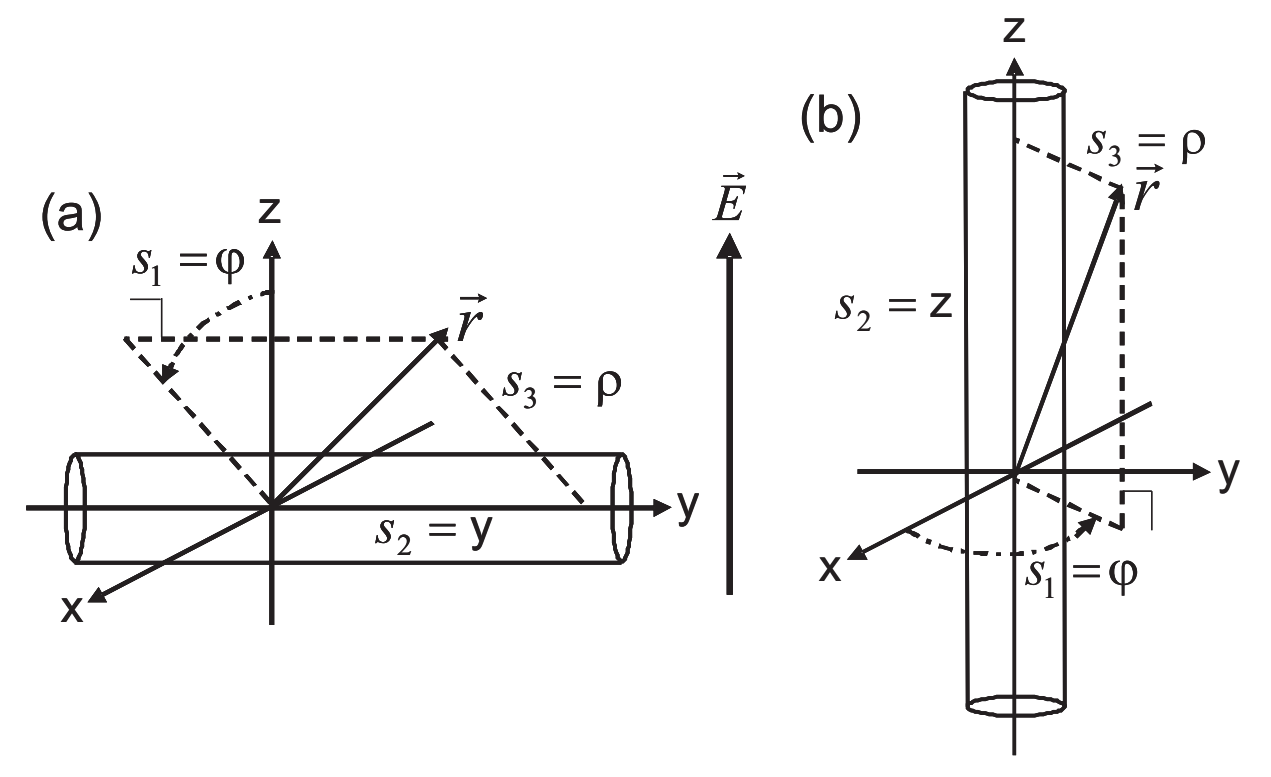

Figure 9: (a) The cylindrical coordinates for parametrization of the sides of a rod for perpendicular orientation. (b) The cylindrical coordinates for parametrization of the sides of a rod for parallel orientation.

$$
\begin{aligned}
& \hat{\mathbf{t}}_{2}=(0,1,0), \\
& \hat{\mathbf{t}}_{3}=(\sin \varphi, 0, \cos \varphi),
\end{aligned}
$$

and,

$$
h_{1}=\rho, h_{2}=1, \quad h_{3}=1 .
$$

For a rod with a high aspect ratio, the charge density is essentially independent of $y$. For long and thin rods the relatively small contributions from the ends can be neglected, which amounts to the approximation that $\sigma$ is only a function of the angle $\varphi$. Substitution of these expressions in the general form (12) leads to eq.(21).

In case of parallel orientation, the standard cylindrical coordinates can be used to parameterize the surface of the rod, with $s_{1}=\varphi, s_{2}=z$ and $s_{3}=\rho$, and $\mathbf{r}=(\rho \cos \{\varphi\}, \rho \sin \{\varphi\}, z)$. Using the definitions as given in Fig.9b, the three basis vectors are,

$$
\begin{aligned}
& \hat{\mathbf{t}}_{1}=(-\sin \varphi, \cos \varphi, 0), \\
& \hat{\mathbf{t}}_{2}=(0,0,1), \\
& \hat{\mathbf{t}}_{3}=(\cos \varphi, \sin \varphi, 0),
\end{aligned}
$$




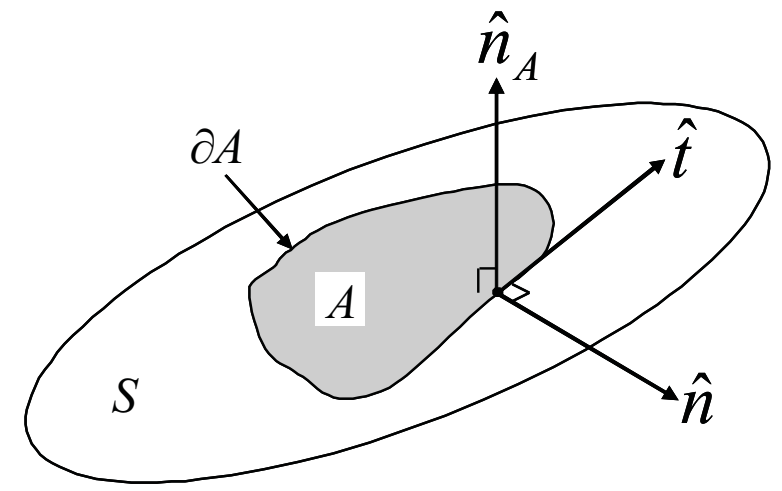

Figure 10: An arbitrary subsurface $\mathcal{A}$ on the surface $\mathcal{S}$ of the core of the colloidal rod. The boundary of $\mathcal{A}$ is a closed curve on $\mathcal{S}$ which is denoted by $\partial \mathcal{A}$. The unit vector $\hat{\mathbf{n}}_{\mathcal{A}}$ is perpendicular to the surface, $\hat{\mathbf{t}}$ is tangential to both the curve $\partial \mathcal{A}$ and $\mathcal{S}$, while $\hat{\mathbf{n}}$ is perpendicular to $\partial \mathcal{A}$ and tangential to $\mathcal{S}$.

and,

$$
h_{1}=\rho, h_{2}=1, h_{3}=1 .
$$

Substitution into the general form (12) of the diffusion equation now immediately leads to eq.(35).

\section{Appendix B: The continuity equation on a surface}

Consider a flux $\mathbf{j}(\mathbf{r})$ that is defined for any position $\mathbf{r}$ in $\Re^{3}$. In this appendix we shall not denote time dependencies for brevity. Let $\mathcal{A}$ be an arbitrary part of a surface $\mathcal{S}$, as sketched in Fig.10. In our case the latter surface is the surface of the colloidal core. The number of ions within $\mathcal{A}$ is equal to the surface integral of $c$ ranging over $\mathcal{A}$,

$$
N_{\mathcal{A}}=\int_{\mathcal{A}} d S c
$$

where $d S$ is an infinitesimal small surface element. Let $\hat{\mathbf{t}}$ denote the unit tangential vector to the boundary $\partial \mathcal{A}$ of $\mathcal{A}$, and $\hat{\mathbf{n}}$ the unit normal pointing outward of $\mathcal{A}$, both of which are tangential to the core's surface $\mathcal{S}$ (see also Fig.10). The orientation of $\hat{\mathbf{t}}$ is such that it points into the anti-clockwise orientation of the curve $\partial \mathcal{A}$. The time-rate of change of the number of ions in $\mathcal{A}$ due to in- and out-flux through the boundary $\partial \mathcal{A}$ is equal to,

$$
\frac{d N_{\mathcal{A}}}{d t}=-\oint_{\partial \mathcal{A}} d l \hat{\mathbf{n}} \cdot \mathbf{j}
$$


where $\mathbf{j}$ is understood to be tangential to the surface $\mathcal{S}$. Since, $\hat{\mathbf{n}}=\hat{\mathbf{t}} \times \hat{\mathbf{n}}_{\mathcal{A}}$, where $\hat{\mathbf{n}}_{\mathcal{A}}$ is the unit normal on the volume that is bounded by the surface $\mathcal{S}$ (see again Fig.10), it follows that,

$$
\frac{d N_{\mathcal{A}}}{d t}=-\oint_{\partial \mathcal{A}} d l\left(\hat{\mathbf{t}} \times \hat{\mathbf{n}}_{\mathcal{A}}\right) \cdot \mathbf{j}=-\oint_{\partial \mathcal{A}} d \mathbf{l} \cdot\left(\hat{\mathbf{n}}_{\mathcal{A}} \times \mathbf{j}\right)
$$

where $d \mathbf{l}=\hat{\mathbf{t}} d l$. From Stokes's theorem and eq.(88) is follows that,

$$
\int_{\mathcal{A}} d S\left\{\frac{\partial c}{\partial t}+\hat{\mathbf{n}}_{\mathcal{A}} \cdot\left[\nabla \times\left(\hat{\mathbf{n}}_{\mathcal{A}} \times \mathbf{j}\right)\right]\right\}=0 .
$$

Since this holds for any arbitrary integration range (embedded in $\mathcal{S}$ ), it follows that, assuming continuity,

$$
\frac{\partial c}{\partial t}+\hat{\mathbf{n}}_{\mathcal{A}} \cdot\left[\nabla \times\left(\hat{\mathbf{n}}_{\mathcal{A}} \times \mathbf{j}\right)\right]=0 .
$$

Since,

$$
\nabla \times\left(\hat{\mathbf{n}}_{\mathcal{A}} \times \mathbf{j}\right)=\hat{\mathbf{n}}_{\mathcal{A}} \nabla \cdot \mathbf{j}-\mathbf{j} \nabla \cdot \hat{\mathbf{n}}_{\mathcal{A}}+(\mathbf{j} \cdot \nabla) \hat{\mathbf{n}}_{\mathcal{A}}-\left(\hat{\mathbf{n}}_{\mathcal{A}} \cdot \nabla\right) \mathbf{j}
$$

while

$$
\begin{aligned}
\hat{\mathbf{n}}_{\mathcal{A}} \cdot\left[\mathbf{j} \nabla \cdot \hat{\mathbf{n}}_{\mathcal{A}}\right] & =0, \\
\hat{\mathbf{n}}_{\mathcal{A}} \cdot\left[(\mathbf{j} \cdot \nabla) \hat{\mathbf{n}}_{\mathcal{A}}\right] & =0,
\end{aligned}
$$

it follows that,

$$
\frac{\partial c}{\partial t}=-\nabla \cdot \mathbf{j}+\hat{\mathbf{n}}_{\mathcal{A}} \hat{\mathbf{n}}_{\mathcal{A}}: \nabla \mathbf{j}=-\left[\hat{\mathbf{I}}-\hat{\mathbf{n}}_{\mathcal{A}} \hat{\mathbf{n}}_{\mathcal{A}}\right]: \nabla \mathbf{j}
$$

where $\hat{\mathbf{I}}$ is the identity. Using that,

$$
\hat{\mathbf{I}}=\hat{\mathbf{t}}_{1} \hat{\mathbf{t}}_{1}+\hat{\mathbf{t}}_{2} \hat{\mathbf{t}}_{2}+\hat{\mathbf{n}}_{\mathcal{A}} \hat{\mathbf{n}}_{\mathcal{A}}
$$

for any two mutually orthogonal unit vectors which are tangential to $\mathbf{S}$, it is thus found that,

$$
\frac{\partial c}{\partial t}=-\left[\hat{\mathbf{t}}_{1} \hat{\mathbf{t}}_{1}+\hat{\mathbf{t}}_{2} \hat{\mathbf{t}}_{2}\right]: \nabla \mathbf{j} .
$$

Since $\hat{\mathbf{t}}_{1} \hat{\mathbf{t}}_{1}+\hat{\mathbf{t}}_{2} \hat{\mathbf{t}}_{2}$ is the projection operator onto the core surface $\mathcal{S}$, the gradient operator in the above equation can be replaced by the surface-gradient operator $\nabla_{s}$, which leads to the continuity equation,

$$
\frac{\partial c}{\partial t}=-\left[\hat{\mathbf{t}}_{1} \hat{\mathbf{t}}_{1}+\hat{\mathbf{t}}_{2} \hat{\mathbf{t}}_{2}\right]: \nabla_{s} \mathbf{j} .
$$


Substitution of eq.(81) and using orthonormality of the two unit surface vectors leads to,

$$
\frac{\partial c}{\partial t}=-\frac{1}{h_{1}} \hat{\mathbf{t}}_{1} \cdot \frac{\partial \mathbf{j}}{\partial s_{1}}-\frac{1}{h_{2}} \hat{\mathbf{t}}_{2} \cdot \frac{\partial \mathbf{j}}{\partial s_{2}} .
$$

Writing the flux in terms of the two surface unit vectors,

$$
\mathbf{j}=j_{1} \hat{\mathbf{t}}_{1}+j_{2} \hat{\mathbf{t}}_{2},
$$

and using the two identities,

$$
\begin{aligned}
\hat{\mathbf{t}}_{i} \cdot \frac{\partial \hat{\mathbf{t}}_{i}}{\partial s_{j}} & =0, \\
\hat{\mathbf{t}}_{i} \cdot \frac{\partial \hat{\mathbf{t}}_{j}}{\partial s_{i}} & =\frac{1}{h_{j}} \frac{\partial h_{i}}{\partial s_{j}}, \quad i \neq j,
\end{aligned}
$$

the equation of motion (99) is most conveniently rewritten as,

$$
\frac{\partial c}{\partial t}=-\left[\frac{1}{h_{1}} \frac{\partial j_{1}}{\partial s_{1}}+\frac{1}{h_{2}} \frac{\partial j_{2}}{\partial s_{2}}\right]-\frac{1}{h_{1} h_{2}}\left[j_{1} \frac{\partial h_{2}}{\partial s_{1}}+j_{2} \frac{\partial h_{1}}{\partial s_{2}}\right] .
$$

For the specific flux in eq.(6), this leads to the diffusion equation (7).

\section{Appendix C: Derivation of eq.(60-64) for the torque}

The external electric field is decomposed in its component $\mathbf{E}_{\|}=\hat{\mathbf{u}} \hat{\mathbf{u}} \cdot \mathbf{E}$ parallel to the rods orientation and its perpendicular component $\mathbf{E}_{\perp}=[\hat{\mathbf{I}}-\hat{\mathbf{u}} \hat{\mathbf{u}}] \cdot \mathbf{E}$, where $\hat{\mathbf{I}}$ is the identity. Within linear response, the condensate charge can be written as,

$$
\sigma=\sigma_{\|}+\sigma_{\perp}
$$

where $\sigma_{\|, \perp}$ is the charge density that is induced by $\mathbf{E}_{\|, \perp}$. The potential of the double layer is similarly written as,

$$
\Psi_{d l}=\Psi_{\|}+\Psi_{\perp},
$$

with $\Psi_{\|, \perp}$ the potential that is induced by $\mathbf{E}_{\|, \perp}$.

In order to perform the integrations, the two unit vectors $\hat{\mathbf{v}}$ and $\hat{\mathbf{w}}$ are introduced, which define a mutually orthonormal coordinate system with $\hat{\mathbf{u}}$. The surface of the cylinder is now parametrized with the variables $l \in[-L / 2, L / 2]$ and $\varphi \in[0,2 \pi)$,

$$
\mathbf{r}=-a \sin \{\varphi\} \hat{\mathbf{v}}+a \cos \{\varphi\} \hat{\mathbf{w}}+l \hat{\mathbf{u}}
$$


The unit vectors $\hat{\mathbf{v}}, \hat{\mathbf{w}}$ and $\hat{\mathbf{u}}$ form an orthonormal right-handed coordinate system, that is,

$$
\begin{aligned}
\hat{\mathbf{u}} \times \hat{\mathbf{v}} & =\hat{\mathbf{w}}, \\
\hat{\mathbf{w}} \times \hat{\mathbf{u}} & =\hat{\mathbf{v}}, \\
\hat{\mathbf{v}} \times \hat{\mathbf{w}} & =\hat{\mathbf{u}} .
\end{aligned}
$$

where $\hat{\mathbf{v}}$ is perpendicular to the external field,

$$
\hat{\mathbf{v}} \cdot \mathbf{E}(t)=0
$$

The electric field can thus be written as,

$$
\mathbf{E}=\left[E_{0, \perp} \hat{\mathbf{w}}+E_{0, \|} \hat{\mathbf{u}}\right] \exp \{i \omega t\}
$$

where $E_{0, \|}$ and $E_{0, \perp}$ are the amplitudes of the electric field in the direction parallel and perpendicular to the rod, respectively. The variable $\varphi$ is the same that we used before, where polarization for parallel and perpendicular orientation is analyzed. Since $\Psi_{\|}$is a function of $l$ only, and $\Psi_{\perp}$ is a function of $\varphi$, the surface gradient of the double-layer potential is equal to,

$$
\nabla_{s} \Psi(\mathbf{r}, t)=-\frac{1}{a}[\hat{\mathbf{v}} \cos \varphi+\hat{\mathbf{w}} \sin \varphi] \frac{\partial \Psi_{\perp}(\varphi, t)}{\partial \varphi}+\hat{\mathbf{u}} \frac{\partial \Psi_{\|}(l, t)}{\partial l}
$$

Note that the condensate can not exert a torque on itself, so that the total potential can be used instead of just its contribution from the double layer.

Using eqs. $(33,52)$ and $\hat{\mathbf{u}} \times \hat{\mathbf{w}}=-\hat{\mathbf{v}}$, and noting that $\sigma_{\perp} \sim \Psi_{\perp} \sim \cos \{\varphi\}$, while $\sigma_{\|}$and $\Psi_{\|}$are odd functions of $l$, it is found that,

$$
\mathbf{T}=\mathbf{T}_{\|}+\mathbf{T}_{\perp},
$$

where,

$$
\mathbf{T}_{\|}(\hat{\mathbf{u}}, t)=-a \hat{\mathbf{v}} \int_{-L / 2}^{L / 2} d l \int_{0}^{2 \pi} d \varphi l \sigma_{\|}(l, t)\left[\frac{1}{a} \sin \{\varphi\} \frac{\partial \Psi_{\perp}(\varphi, t)}{\partial \varphi}+E_{\perp}(t)\right]
$$

and,

$$
\mathbf{T}_{\perp}(\hat{\mathbf{u}}, t)=-a^{2} \hat{\mathbf{v}} \int_{-L / 2}^{L / 2} d l \int_{0}^{2 \pi} d \varphi \cos \{\varphi\} \sigma_{\perp}(\varphi, t)\left[\frac{\partial \Psi_{\|}(l, t)}{\partial l}-E_{\|}(t)\right]
$$


The torque $\mathbf{T}_{\|}$is the contribution to the total torque due to forces on the parallel charge distribution, while $\mathbf{T}_{\perp}$ is due to forces on the perpendicular charge distribution.

As this point, the time dependencies have to be made explicit. Using that $\mathbf{E}(t)=\mathbf{E}_{0} \cos \{\omega t\}$, and expressing the charge densities and the potentials in terms of the response functions, leads to,

$$
\begin{aligned}
\mathbf{T}_{\|}(\hat{\mathbf{u}}, t)= & -a \hat{\mathbf{v}} \int_{-L / 2}^{L / 2} d l \int_{0}^{2 \pi} d \varphi l\left[R_{\|}^{\prime}(l) \cos \{\omega t\}+R_{\|}^{\prime \prime}(l) \sin \{\omega t\}\right] \\
& \times\left[\frac{1}{a} \sin \{\varphi\}\left(\frac{d P_{\perp}^{\prime}(\varphi)}{d \varphi} \cos \{\omega t\}+\frac{d P_{\perp}^{\prime \prime}(\varphi)}{d \varphi} \sin \{\omega t\}\right)+E_{0, \perp} \cos \{\omega t\}\right],
\end{aligned}
$$

and,

$$
\begin{aligned}
\mathbf{T}_{\perp}(\hat{\mathbf{u}}, t)= & -a^{2} \hat{\mathbf{v}} \int_{-L / 2}^{L / 2} d l \int_{0}^{2 \pi} d \varphi \cos \{\varphi\}\left[R_{\perp}^{\prime}(\varphi) \cos \{\omega t\}+R_{\perp}^{\prime \prime}(\varphi) \sin \{\omega t\}\right] \\
& \times\left[\left(\frac{d P_{\|}^{\prime}(l)}{d l} \cos \{\omega t\}+\frac{d P_{\|}^{\prime \prime}(l)}{d l} \sin \{\omega t\}\right)-E_{0, \|} \cos \{\omega t\}\right],
\end{aligned}
$$

where we used obvious abbreviated notations for the response functions. Here, $\mathbf{E}_{0, \|}$ and $\mathbf{E}_{0, \perp}$ are the parallel and perpendicular components of the field amplitude $\mathbf{E}_{0}$.

For the purpose of calculating the torques, the response functions for parallel orientation are most conveniently written as,

$$
\begin{aligned}
R_{\|}^{\prime}(l) & =\left[K_{\|}^{(-)} \cos \{2 \Omega l / L\} \sinh \{2 \Omega l / L\}+K_{\|}^{(+)} \sin \{2 \Omega l / L\} \cosh \{2 \Omega l / L\}\right] E_{0, \|}, \\
R_{\|}^{\prime \prime}(l) & =\left[K_{\|}^{(+)} \cos \{2 \Omega l / L\} \sinh \{2 \Omega l / L\}-K_{\|}^{(-)} \sin \{2 \Omega l / L\} \cosh \{2 \Omega l / L\}\right] E_{0, \|}, \\
P_{\|}^{\prime}(l) & =\frac{a}{\epsilon} K(\kappa a) R_{\|}^{\prime}(l), \\
P_{\|}^{\prime \prime}(l) & =\frac{a}{\epsilon} K(\kappa a) R_{\|}^{\prime \prime}(l),
\end{aligned}
$$

where,

$$
K_{\|}^{( \pm)}=\frac{\epsilon \kappa_{c} L}{1+2 \kappa_{c} a K(\kappa a)} F^{( \pm)}(\Omega)
$$

The response functions for perpendicular orientation are written as,

$$
\begin{aligned}
R_{\perp}^{\prime}(\varphi) & =K_{\perp}^{\prime} \cos \{\varphi\} E_{0, \perp}, \\
R_{\perp}^{\prime \prime}(\varphi) & =K_{\perp}^{\prime \prime} \cos \{\varphi\} E_{0, \perp}, \\
P_{\perp}^{\prime}(\varphi) & =\frac{a}{2 \epsilon} R_{\perp}^{\prime}(\varphi), \\
P_{\perp}^{\prime \prime}(\varphi) & =\frac{a}{2 \epsilon} R_{\perp}^{\prime \prime}(\varphi),
\end{aligned}
$$


where,

$$
\begin{aligned}
K_{\perp}^{\prime} & =\frac{2 \epsilon \kappa_{c} a\left(1+\kappa_{c} a B(\kappa a)\right)}{\left(1+\kappa_{c} a B(\kappa a)\right)^{2}+\Lambda_{\perp}^{2}} \\
K_{\perp}^{\prime \prime} & =\frac{2 \epsilon \kappa_{c} a \Lambda_{\perp}}{\left(1+\kappa_{c} a B(\kappa a)\right)^{2}+\Lambda_{\perp}^{2}} .
\end{aligned}
$$

Using the identities,

$$
\begin{aligned}
& \int_{-L / 2}^{L / 2} d l l \cos \{2 \Omega l / L\} \sinh \{2 \Omega l / L\}= \\
& \quad \frac{L^{2}}{4} \frac{1}{\Omega^{2}}[-\sin \{\Omega\} \cosh \{\Omega\}+\Omega \cos \{\Omega\} \cosh \{\Omega\}+\Omega \sin \{\Omega\} \sinh \{\Omega\}]
\end{aligned}
$$

and,

$$
\begin{aligned}
& \int_{-L / 2}^{L / 2} d l l \sin \{2 \Omega l / L\} \cosh \{2 \Omega l / L\}= \\
& \quad \frac{L^{2}}{4} \frac{1}{\Omega^{2}}[\cos \{\Omega\} \sinh \{\Omega\}+\Omega \sin \{\Omega\} \sinh \{\Omega\}-\Omega \cos \{\Omega\} \cosh \{\Omega\}],
\end{aligned}
$$

a long but straightforward calculation finally leads to,

$$
\begin{gathered}
\mathbf{T}_{\|}(\hat{\mathbf{u}}, t)=-\frac{\pi}{4} L^{3} \hat{\mathbf{v}} E_{0, \perp} E_{0, \|} \frac{\epsilon \kappa_{c} a}{\left(1+\kappa_{c} a B(\kappa a)\right)^{2}+\Lambda_{\perp}^{2}} \frac{1}{1+2 \kappa_{c} a K(\kappa a)} \\
\times\left[I^{(-)}(\Omega) \cos \{\omega t\}+\left(\Omega^{-2}-I^{(+)}(\Omega)\right) \sin \{\omega t\}\right] \\
\times\left[\left\{2\left(1+\kappa_{c} a B(\kappa a)\right)^{2}-\kappa_{c} a\left(1+\kappa_{c} a B(\kappa a)\right)+2 \Lambda_{\perp}^{2}\right\} \cos \{\omega t\}\right. \\
\left.-\kappa_{c} a \Lambda_{\perp} \sin \{\omega t\}\right]
\end{gathered}
$$

and,

$$
\begin{aligned}
\mathbf{T}_{\perp}(\hat{\mathbf{u}}, t)= & \frac{\pi}{2} \frac{L^{3}}{p^{2}} \hat{\mathbf{v}} E_{0, \perp} E_{0, \|} \frac{\epsilon \kappa_{c} a}{\left(1+\kappa_{c} a B(\kappa a)\right)^{2}+\Lambda_{\perp}^{2}} \\
& \times\left[\cos \{\omega t\}-\frac{2 \kappa_{c} a K(\kappa a)}{1+2 \kappa_{c} a K(\kappa a)} \Omega^{2}\left(I^{(+)}(\Omega) \cos \{\omega t\}+I^{(-)}(\Omega) \sin \{\omega t\}\right)\right] \\
& \times\left[\left(1+\kappa_{c} a B(\kappa a)\right) \cos \{\omega t\}+\Lambda_{\perp} \sin \{\omega t\}\right],
\end{aligned}
$$

where $I^{( \pm)}(\Omega)$ is given in eq. (64).

From the definitions of $\mathbf{E}_{0, \|}$ and $\mathbf{E}_{0, \perp}$ in terms of $\hat{\mathbf{u}}$ as given in the beginning of this appendix, it is readily found that $E_{0, \|} E_{0, \perp}=E_{0}^{2}|\sin \{\gamma\} \cos \{\gamma\}|$, where $\gamma$ is the angle between $\mathbf{E}_{0}$ and $\hat{\mathbf{u}}$ (the 
absolute value renders this expression independent on whether the smallest or largest angle between $\mathbf{E}_{0}$ and $\hat{\mathbf{u}}$ is used). Since $|\sin \gamma|=\left|\hat{\mathbf{E}}_{0} \times \hat{\mathbf{u}}\right|$ and $|\cos \gamma|=\left|\hat{\mathbf{E}}_{0} \cdot \hat{\mathbf{u}}\right|$, while $\hat{\mathbf{v}}$ is in the direction of $\hat{\mathbf{E}}_{0} \times \hat{\mathbf{u}}$, with $\hat{\mathbf{E}}_{0}$ the unit vector along $\mathbf{E}_{0}$, this finally leads to eqs. $(61,62)$ for the two torques.

\section{Appendix D: The full expression for the orientational or- der parameter}

From substitution of eq.(72) for $T$ into eq.(74) it follows that,

$$
Q_{E}(t)=Q_{\|}(t)+Q_{\perp}(t)
$$

with,

$$
\begin{aligned}
Q_{\|}(t)= & \frac{\pi \beta D_{r} E_{0}^{2} L^{3} \epsilon}{10\left[\left(6 D_{r}\right)^{2}+(2 \omega)^{2}\right]} \\
\times & \left\{F_{1} F_{3}\left[\frac{\omega^{2}}{3 D_{r}}+\cos \{\omega t\}\left(6 D_{r} \cos \{\omega t\}+2 \omega \sin \{\omega t\}\right)\right]\right. \\
& +F_{2} F_{4}\left[\frac{\omega^{2}}{3 D_{r}}+\sin \{\omega t\}\left(6 D_{r} \sin \{\omega t\}-2 \omega \cos \{\omega t\}\right)\right] \\
& \left.+\frac{1}{2}\left(F_{1} F_{4}+F_{2} F_{3}\right)\left(6 D_{r} \sin \{2 \omega t\}-2 \omega \cos \{2 \omega t\}\right)\right\},
\end{aligned}
$$

and,

$$
\begin{aligned}
Q_{\perp}(t)=- & \frac{\pi \beta D_{r} E_{0}^{2} L^{3} \epsilon}{5 p^{2}\left[\left(6 D_{r}\right)^{2}+(2 \omega)^{2}\right]} \\
\times & \left\{H_{1} H_{3}\left[\frac{\omega^{2}}{3 D_{r}}+\cos \{\omega t\}\left(6 D_{r} \cos \{\omega t\}+2 \omega \sin \{\omega t\}\right)\right]\right. \\
& +H_{2} H_{4}\left[\frac{\omega^{2}}{3 D_{r}}+\sin \{\omega t\}\left(6 D_{r} \sin \{\omega t\}-2 \omega \cos \{\omega t\}\right)\right] \\
& \left.+\frac{1}{2}\left(H_{1} H_{4}+H_{2} H_{3}\right)\left(6 D_{r} \sin \{2 \omega t\}-2 \omega \cos \{2 \omega t\}\right)\right\} .
\end{aligned}
$$

where, as before, the indices $\|$ and $\perp$ refer to the contributions arising from the torque on the charge distribution along and perpendicular to the rod's long axis, respectively.

This expression is valid for all frequencies. The largest time scale appearing in these expressions is the time $1 / D_{r}$ for the rod to rotate (for fd this is about $0.05 s$ ). This time is very much larger than the times $a^{2} / D$ and $L^{2} / 4 D^{\mathrm{eff}}$ that condensed ions need to give rise to polarization in the perpendicular and parallel direction, respectively. 


\section{References}

[1] H. Kramer, C. Graf, M. Hagenbüchle, C. Johner, C. Martin, P. Schwind, R. Weber, J. Phys. II 4, $1061(1994)$.

[2] K. Kang, J.K.G. Dhont, Eur. Phys. Lett. 84, 14005 (2008).

[3] K. Kang, J.K.G. Dhont, Soft Matter 6, 273 (2010).

[4] K. Kang, J.K.G. Dhont, Eur. Phys. J. E 30, 333 (2009).

[5] F. Mantegazza, M. Caggioni, M.L. Jiménez, T. Bellini, Nature Physics 1, 103 (2005).

[6] J.P. Singh, P.P. Lele, F. Nettesheim, N.J. Wagner, E.M. Furst, Phys. Rev. E 79, 050401 (2009).

[7] J.K.G. Dhont, K. Kang, Eur. Phys. J. E 33, 51-68 (2010).

[8] M. Mandel, Mol. Phys. 4, 489 (1961).

[9] G.S. Manning, J. Chem. Phys. 51, 924 (1969).

[10] G.S. Manning, J. Chem. Phys. 51, 3249 (1969).

[11] F. Oosawa, Polyelectrolytes (Marcel Dekker, New York, 1971).

[12] F. van der Touw, M. Mandel, Biophys. Chem. 2, 218 (1974).

[13] G.S. Manning, Biophys. Chem. 9, 65 (1978).

[14] G.S. Manning, Q. Rev. Biophys. 11, 179 (1978).

[15] G.S. Manning, J. Chem. Phys. 99, 477 (1993).

[16] G.S. Manning, J. Ray, Langmuir 10, 962 (1994).

[17] G.S. Manning, Macromolecules 40, 8071 (2007).

[18] G.S. Manning, Macromolecules 41, 6217 (2008).

[19] G.S. Manning, this issue.

[20] S. Takashima, Adv. Chem. Ser. 63, 232 (1967).

[21] S. Takashima, Electrical properties of biopolymers and membranes, page 204, Adam Hilger, Bristol and Philadelphia (1989). 
[22] M. A. Young, B. Jayaram, D. L. Beveridge, J. Am. Chem. Soc. 119, 59 (1997).

[23] S. Y. Ponomarev, K. M. Thayer, D. L. Beveridge, Proc. Natl. Acad. Sci. USA 101, 14771 (2004).

[24] Personal communication with G.S. Manning.

[25] M. Guéron, G. Weisbuch, Biopolymers 19, 353 (1980).

[26] M. le Bret, H. Zimm, Biopolymers 23, 287 (1984).

[27] M. Deserno, C. Holm, S. May, Macromolecules 33, 199 (2000).

[28] B. O'Shaughnessy, Q. Yang, Phys. Rev. Lett. 94, 048301 (2005).

[29] D. Ben-Yaakov, D. Andelman, D. Harries, R. Podgornik, J. Chem. Phys. B 113, 6001 (2009).

[30] R. Okamoto, A. Onuki, J. Chem. Phys. 131, 094905 (2009).

[31] B.A. Todd, D.C. Rau, Nucleic Acids Research 36, 501 (2008).

[32] A. Chepelianskii, F. Mohammad-Rafiee, E. Trizac, E. Raphaël, J. Phys. Chem. B, 113, 3743 (2009).

[33] J.-M. Y. Carrillo, A.V. Dobrynin, J. Phys. Chem. B, 1149391 (2010).

[34] M. Luksic, B. Hribar-Lee, R. Buchner, V. Vlachy, Phys. Chem. Chem. Phys. 11, 10053 (2009).

[35] R. Buchner, G. Hefter, Phys. Chem. Chem. Phys. 11, 8984 (2009).

[36] J. Ray, G.S. Manning, Langmuir 10, 2450 (1994).

[37] K.R. Purdy, Z. Dogic, S. Fraden, A. Rühm, L. Lurio, S.G.J. Mochrie, Phys. Rev. E. 67, 031708 (2003).

[38] J. Torbet, G. Maret, Biopolymers 20, 2657 (1981).

[39] K. Kang, A. Wilk, A. Patkowski, J.K.G. Dhont, J. Chem. Phys. 126, 214501 (2007). 